\title{
Anatomía de la dispersión urbana en Barcelona
}

Iván Muñiz. Universitat Autònoma de Barcelona, Barcelona, España.

Miquel-Àngel García-López. Universitat Autònoma de Barcelona - Institut d'Economia de Barcelona, Barcelona, España.

RESUMEN | No existe una definición comúnmente aceptada de dispersión urbana (urban sprawl), de modo que la investigación aplicada ha trabajado con diferentes indicadores que pretenden a su vez capturar los distintos significados con que se ha dotado al término. En este trabajo se mide cómo ha evolucionado la dispersión de la población y del empleo de la Región Metropolitana de Barcelona (RMB) entre 1986 y 2001. Las dimensiones exploradas se dividen en dos grupos: i) forma urbana (desconcentración, baja densidad y discontinuidad) y ii) estructura urbana. A diferencia de algunos trabajos donde el policentrismo y el crecimiento lineal se presentan como dos formas de dispersión, en nuestro caso contraponemos la idea de expansión urbana estructurada (lineal o policéntrica) con la de expansión urbana desestructurada (amorfa, caótica o aleatoria), equiparando esta última con la cuarta dimensión de la dispersión. Los resultados obtenidos indican que la población y el empleo de la RMB se han descentralizado de forma desconcentrada y la densidad residencial se ha reducido. Sin embargo, la RMB no ha crecido de forma discontinua ni desestructurada, sino todo lo contrario.

PALABRAS CLAVE | estructura urbana, expansión urbana, morfología urbana

ABSTRACT | There is no commonly accepted definition of urban sprawl, and therefore applied research has worked with different indicators aiming to grasp the diverse meanings assigned to the term. This study measures how the dispersion of population and employment in the Barcelona Metropolitan Region (BMR) developed between 1986 and 2001. The dimensions that were investigated are divided into two groups. The first one involves dimensions of urban form (decentralization, low density and discontinuity), and the second, of urban structure. As opposed to some studies, where polycentrism and linear growth are presented as two forms of sprawl, in our case we contrast the idea of structured urban expansion (linear or poly-centric) with de structured urban expansion (amorphous, chaotic or random), identifying the latter with the fourth dimension of sprawl. The results suggest that population and employment in the BMR has become decentralized in a non-concentrated way and, in the case of population, the density has decreased. However, the BMR has not grown in a discontinuous or de-structured way, but quite the contrary.

KEY WORDS | urban structure, urban sprawl, urban morphology 


\section{Introducción}

Aunque con algunas excepciones (Gordon \& Richardson, 1997; Persky \& Wiewel, 2000), existe un elevado consenso sobre los costes que comporta la dispersión urbana. Desde un punto de vista social, cabe destacar los problemas de segregación y limitada movilidad de los colectivos con menor renta (Burchell et al., 1998; Power 2001; Downs, 1999). En cuanto a los efectos ambientales, la dispersión puede traducirse en la ocupación de espacios de gran valor agrícola, ecológico o paisajístico. Además, dificulta la oferta de un transporte público competitivo, por lo que tiende a imponerse un modelo de movilidad basado en el automóvil, que genera grandes emisiones de CO2 (Newman \& Kenworthy, 1989; Kahn, 2000; Camagni, Gibelli \& Rigamonti., 2002; Muñiz \& Galindo, 2005). La dispersión urbana no solo tiene impactos sociales y ambientales, sino que es un modelo de ocupación del suelo económicamente ineficiente, ya que los promotores inmobiliarios no internalizan los costes públicos y externalidades propias de un modelo residencial de baja densidad (Anas, Arnott \& Small, 1998; Brueckner, 2000; Wassmer, 2001).

En contraste con el relativo consenso sobre su carácter nocivo, no existe una única definición de dispersión urbana. En general, frente a un enfoque típicamente estadounidense, donde el término 'dispersión urbana' (urban sprawl) suele equipararse con cualesquiera de las formas que pueda adoptar el crecimiento de una ciudad, en Europa tiende a utilizarse de forma más acotada, con énfasis en el carácter caótico y aleatorio del modelo de ocupación del suelo (European Environment Agency, 2006). Esta diversidad de definiciones se ha visto acompañada de diferentes estrategias de medición. La gran variedad de indicadores propuestos denota la falta de una estrategia cuantitativa mínimamente coordinada.

Mediante una batería de indicadores, esta investigación pretende capturar la dispersión que se ha dado en la Región Metropolitana de Barcelona (RMB) entre 1986 y 2001. A diferencia de la mayoría de trabajos, centrados principalmente en la ocupación de suelo para fines residenciales, se cuantifican las diferentes dimensiones de la dispersión, tanto de la población como del empleo. La investigación se ha orientado hacia un enfoque híbrido entre el norteamericano (multidimensional) y el europeo (centrado en la idea de desestructuración del espacio). Debido a que cualquier proceso de esta naturaleza no se da en un vacío institucional, sino que viene afectado por las políticas de suelo, vivienda y dotación de infraestructuras, se ha incluido un apartado donde se señalan las posibles causas del elevado consumo de suelo para fines urbanos que se ha dado España durante el periodo estudiado y que también parece haberse trasladado a la RMB. Los principales resultados obtenidos indican que el suelo urbanizado ha aumentado de forma significativa, pero que, aun así, el peso de la vivienda unifamiliar no se ha incrementado, ni la metrópoli se ha extendido de forma discontinua o desestructurada, sino articulada a partir de los principales accesos a la red viaria y los subcentros de población y empleo. 


\section{La forma y estructura de la dispersión urbana}

\section{Definición}

El término urban sprawl ha demostrado ser extremadamente elástico, utilizándose para caracterizar fenómenos de expansión urbana de muy diferente naturaleza (Audirac, Shermyen \& Smith., 1990; Kirby, 2000). A continuación se presenta una muestra de las diversas aproximaciones a la noción de 'dispersión urbana':

a) Como suma exhaustiva de dimensiones descriptivas. El ejemplo más claro es la definición de Galster, Hanson, Ratcliffe, Wolman y Freihage (2001)․ Se trata de una definición multidimensional y no jerarquizada que pretende abarcar todos los modelos que puede seguir un proceso de expansión urbana. Otros trabajos que han seguido una línea similar son los de Torrens y Alberti (2000); Hess et al. (2001); Malpezzi y Guo (2001); Wolman, Galster, Hanson, Ratcliffe y Furdell (2002); Lopez y Hynes (2003); y Angel, Parent y Civco (2007).

b) Dispersión urbana como equivalente a suburbanización. La dispersión se presenta como sinónimo de suburbanización, aunque añadiendo un acento peyorativo (Gordon \& Wong, 1985; Glaeser \& Kahn, 2004; Couch, Karecha, Nuissl \& Rink, 2005; Wassmer \& Edwards, 2005). En este caso suele utilizarse un único indicador.

c) Dispersión urbana como equivalente a contraurbanización. Según el célebre esquema de Berry (1976) sobre las fases del ciclo de vida urbano, la contraurbanización sería el periodo previo a la reurbanización y estaría caracterizado por el crecimiento de la población en las ciudades de tamaño medio y pequeño (zonas rurales) y la caída de población de las grandes. Para muchos, dicha equiparación resulta engañosa en el ámbito europeo, pues la delimitación municipal utilizada en la mayoría de casos - y que superficialmente parecía indicar la decadencia de la gran ciudad- esconde lo que realmente estaba aconteciendo: un intenso proceso de integración metropolitana o metropolización que ha permitido incorporar un buen número de ciudades medianas y pequeñas en el radio de acción de una gran ciudad. No obstante, autores como Chin (2002) la siguen defendiendo.

d) Dispersión urbana como proceso básicamente nocivo. Algunos trabajos se han centrado principalmente en estudiar los impactos negativos de la dispersión, hasta el punto de incluir algunos de ellos en la propia definición (Ewing, 1997).

e) Dispersión urbana como consumo excesivo de suelo. En ocasiones se ha identificado la dispersión urbana con consumo excesivo de suelo, entendiendo por "excesivo" un ritmo de urbanización que supera la tasa de crecimiento de la población (Downs, 1999; Ewing, 1997; Kasanko et al., 2006; European Environment Agency, 2002a, 2006). Los economistas habitualmente dan un significado diferente a la idea de "consumo excesivo", equiparándolo con una ocupación de suelo por encima de lo que resultaría socialmente eficiente, debido a la existencia de fallos de mercado (Brueckner \&

1 "Sprawl is a pattern of land use in an urbanised area that exhibits low levels of some combination of eight distinct dimensions: density, continuity, concentration, clustering, centrality, nuclearity, mixed uses and proximity". Galster et al. (2001), p. 685 . 
Fansler, 1983; Brueckner, 1997, 2000; Wassmer, 2000). Aunque la idea es interesante - especialmente para los economistas - , resulta difícil de cuantificar. Se trata de una herramienta de naturaleza teórica que induce a una pertinente reflexión sobre las limitaciones del libre mercado para conseguir un uso eficiente del territorio.

f) Dispersión urbana como proceso contrario al policentrismo. En contraste con el primer tipo de definición, donde el policentrismo suele aparecer como una de las formas que puede adoptar la dispersión, en Europa el policentrismo tiende a entenderse como un modelo de crecimiento alternativo a la dispersión. La construcción de new towns, villes nouvelles o ciudades satélite ha sido el instrumento de una política que pretende evitar los problemas de la expansión urbana en forma de mancha de aceite (Hall, 1996; Gravagnuolo, 1998; Frey, 1999; Rogers \& Gumuchdjian, 2000). Caracterizar la dispersión como un modelo espacial contrario al policentrismo también ha sido muy común en los trabajos que se han dedicado a examinar la pauta espacial que sigue el empleo descentralizado (Gordon \& Richardson, 1996; Pfister, Freestone \& Murphy, 2000; Coffey \& Shearmur, 2002; Shearmur \& Alvergne, 2003; Lee, 2007).

g) Dispersión urbana como difusión de servicios urbanos. Francesco Indovina (1990) ha popularizado el término città diffusa para caracterizar el salto cualitativo que se ha dado en algunas regiones conformadas por ciudades de tamaño pequeño al adoptar funciones y modelos de vida propios de una gran urbe - sin haber cambiado sustantivamente su morfología - gracias a la creciente integración del territorio ${ }^{2}$.

h) Dispersión urbana como espacio desestructurado. Ya en 1958, W. H. Whyte mostraba su preocupación por el crecimiento amorfo de las ciudades americanas: "The problem is the pattern of growth or, rather, the lack of one" (p. 116), señalaba. Esta identificación de dispersión con expansión aleatoria o amorfa ha sido destacada, entre otros, por Dear y Flusty (1998), Lang y Lefurgy (2003), o Anderson y Bogart (2001) al estudiar las nuevas pautas de localización del empleo. La European Environment Agency (2006) adopta un enfoque similar, aunque no limitado al caso del empleo, sino a la ocupación de suelo para usos urbanos en general. La idea sobre la que se asienta este enfoque es que la mejora en los transportes y las telecomunicaciones ha relajado las restricciones de localización. Si la distancia no importa, o cada vez importa menos, la localización de los agentes vendrá menos condicionada por su proximidad a los elementos que tradicionalmente han estructurado el territorio, como son los centros de empleo o las infraestructuras de transporte (Pressman, 1985; Castells, 1989; Fishman, 1998; Cairncross, 1997).

\section{Nuestra propuesta}

En este trabajo definimos la dispersión como un proceso de expansión urbana que implica la descentralización de la población y del empleo siguiendo un modelo: i) desconcentrado, ii) poco denso, iii) discontinuo y iv) falto de estructura, aleatorio o caótico.

Esta manera de abordar lo que sucede en el margen tiene una gran similitud con los trabajos sobre periurbanización. Véase Allen (2003) o Goffete-Nagot (2000) para una versión actualizada del tema. 
FIGURA 1 Dimensiones de la dispersión urbana

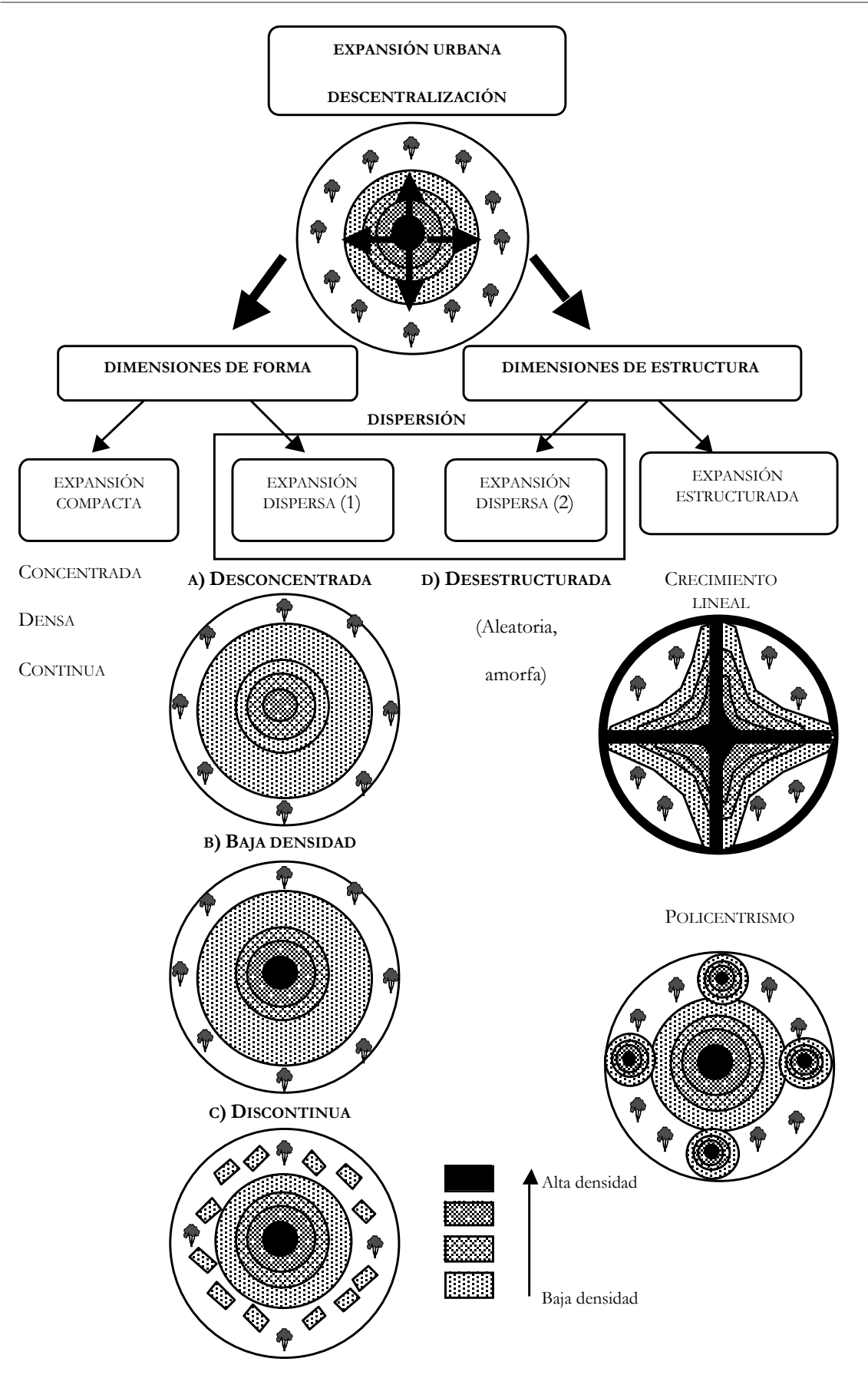

FUENTE ELABORACIÓN PROPIA. 


\section{Las causas del elevado consumo de suelo en España}

Según la European Environment Agency (2006), entre 1990 y 2000 España estuvo en el tercer lugar de los países con la tasa de crecimiento de ocupación de suelo más alta de la Unión Europea, solo por debajo de Irlanda y Portugal. El Instituto Geográfico Nacional midió el crecimiento de la superficie edificada entre 1987 y 2000 (Proyecto Corine Land Cover, Ministerio de Fomento, 2006), confirmando la importante pérdida de suelo agrícola y forestal en beneficio del espacio construido. En conjunto, el suelo urbanizado aumentó $29,5 \%$, siendo el sector residencial el principal responsable de dicho crecimiento.

Entre las causas que han llevado a un espectacular incremento en el número de viviendas en España, cabe destacar las siguientes: i) tipos de interés anormalmente bajos (Trilla, 2001); ii) caída en el tamaño de los hogares (Trilla, 2001), iii) aumento en el número de segundas residencias (Trilla, 2001), iv) cambio de destino de una parte relevante del ahorro familiar colocado en bolsa hacia la compra de vivienda, debido al "pinchazo" - la caída repentina- de los valores tecnológicos y a los bajos tipos de interés bancarios (Naredo, 1996; Balsameda, San Martín \& Sebastián., 2002); y v) el blanqueo de capitales (Naredo, Carpintero \& Marcos, 2003).

Razones de diversa índole explican el hecho de que el crecimiento del sector residencial se haya visto acompañado de una ocupación del suelo tan elevada. En primer lugar, cabe destacar aquellos fenómenos de carácter más genéricos. El aumento de la renta per cápita, el cambio de preferencias de las familias por lugares con un mayor contacto con la naturaleza y una tipología de vivienda unifamilar (Peiser, 1990; Breslaw, 1990; Audirac et al., 1990), junto con la mejora en los transportes y comunicaciones, entrarían en esta categoría (Graham \& Marvin, 2006; Beavestock, Smith \& Taylor, 2000; Wheller, Aoyama \&Warf, 2000).

Hay también causas que obedecen a procesos que actúan a escala europea. Así, por ejemplo, las negociaciones para la liberalización del sector exterior en el marco de la Organización Mundial del Comercio han situado a Europa en un lugar incómodo debido a la política proteccionista aplicada al sector agrícola. Resulta complicado convencer a los países de fuera de la Unión que ven restringidas sus posibilidades de exportar estos productos, para que abran sus fronteras a los bienes y servicios europeos con un alto valor añadido y contenido tecnológico. Es más que probable que todo el sistema de protecciones se vea gradualmente desmantelado, ya que de otro modo se limitaría el desarrollo de aquellos sectores donde Europa demuestra tener una ventaja comparativa, con lo que el mercado podría estar ya descontándolo. Esto otorgaría al suelo agrícola que rodea las ciudades un valor potencial para usos urbanos superior a la capitalización de su rendimiento agrícola, generando una fuerte presión para su recalificación debido a la necesidad de ofrecer alternativas a los agricultores ante un futuro tan incierto (European Environment Agency, 1999, 2002b, 2006; Muñiz, Calatayud \& Garcia-López, 2006). También puede haber tenido un papel importante la elevada fragmentación administrati- 
va que se da en España. Al igual que en Francia, Portugal o Grecia, el sistema de planificación territorial de España es de tipo napoleónico, caracterizado por una elevada autonomía de la administración local (ayuntamientos) para fijar usos del suelo (Newman \& Thomley, 1996). En no pocos casos, los municipios periféricos compiten entre ellos para atraer a la población con mayor capacidad económica ofreciendo vivienda unifamiliar de calidad media y alta, lo que supone un elevado consumo de suelo (Razin \& Rosentraub, 2000; Solé \& Viladecans, 2007).

Por último, algunas de las causas que han llevado a esta importante ocupación de suelo responden a aspectos propios de la realidad española, como por ejemplo la debilidad endémica de las finanzas locales. Colocar suelo en el mercado ha sido -y sigue siendo - una importante fuente de ingresos de los ayuntamientos. Se trata de un mecanismo perverso y miope, debido a que el aumento de la recaudación que se da a corto plazo está por debajo del previsible crecimiento del gasto social en servicios que habrá de cubrir la administración local, pero también la autonómica y la estatal. Tal como apuntan diversos estudios, el coste per cápita que supone la dotación de infraestructuras de transporte, suministro de gas y electricidad, recogida de basuras, o educación y asistencia médica de proximidad, es sensiblemente superior en entornos dispersos que en compactos (Carruthers \& Ulfarsson, 2003; Burchfield, Overman, Puga \& Turner, 2002). También la política de vivienda seguida por el Partido Popular ha tenido un papel fundamental. A pesar de que el número de viviendas ha crecido exponencialmente durante los últimos años, la demanda ha aumentado con tal intensidad que los precios se han disparado, creando una burbuja inmobiliaria. El capital especulativo atraído por unos precios al alza ha alimentado dicho proceso hasta una sobrevaloración de los inmuebles que diferentes fuentes (The Economist, Organización para la Cooperación y Desarrollo Económicos, Fondo Monetario Internacional, Banco Central Europeo) sitúan alrededor del 30\%. La política del Partido Popular para solucionar el problema del precio de la vivienda se centró exclusivamente en relajar las restricciones de oferta de suelo. El precio crecía, se diagnosticó, porque los ayuntamientos ofrecían poco suelo. En otras palabras, los ayuntamientos eran los principales especuladores y culpables del aumento del precio de la vivienda, de modo que para neutralizar su poder se aprobó una nueva Ley de Suelo (Ley de Suelo de 1998) donde, a no ser que se protegiera de forma explícita, todo el suelo pasaba a ser urbanizable. Un diagnóstico tan errado como creer que el precio de la vivienda es caro porque el suelo es caro, en lugar de entender que el suelo es caro porque la vivienda es cara, llevó a una ocupación de suelo a un ritmo desconocido, sin que los precios se estabilizaran. Lo más lógico hubiera sido controlar el flujo de capitales especulativos que alimentaban una demanda desbocada. Hoy, parece claro que las políticas de oferta basadas en la liberalización del suelo fracasaron de forma estrepitosa.

La mayoría de estos argumentos han aparecido publicados — con mayor o menor énfasis - en diferentes trabajos a partir de 2006 (Muñiz et al., 2006; Fernández Durán, 2006; Iglesias, 2007; Jiménez, 2008; Burriel, 2008; Gaja, 2008, Romero, 
2009). En la actualidad existe un amplio consenso en el ámbito académico español sobre la relevancia de la suma de prejuicios con que el reciente modelo de desarrollo ha manejado la cultura del territorio y del urbanismo.

\section{La Región Metropolitana de Barcelona}

\section{Características generales}

La Región Metropolitana de Barcelona está formada por 164 municipios que ocupan una extensión de suelo de unos $3.200 \mathrm{~km}^{2}$ en un radio aproximado de $55 \mathrm{~km}$. Este territorio se corresponde con la definición de "Ámbito Metropolitano" que se establece en el Plan General Territorial de Catalunya (1995), que pretende superar la anterior división provincial de la misma. Contiene una ciudad central de más de un millón y medio de habitantes, el municipio de Barcelona, que es un centro diverso y compacto; y una comarca central que incluye, además del municipio de Barcelona, otros municipios adyacentes. En estos convergen seis comarcas 3 en forma de cuña, cada una de ellas con un municipio central - capital de comarca-, cuatro de las cuales son ciudades de tamaño medio con más de mil años de historia, mientras que las otras dos se han desarrollado recientemente ${ }^{4}$. Contigua al municipio de Barcelona, la primera corona metropolitana se caracteriza por su elevada densidad y la presencia de polígonos de vivienda (conjuntos residenciales de elevada densidad constituido por bloques aislados, similares a las grands ensembles francesas). A continuación se encuentra una segunda corona residencial menos densa que, en algunos casos, concentra elevadas rentas. Por último, alrededor de las capitales de comarca hay extensas áreas que combinan usos residenciales de baja densidad con otros asociados a un mundo rural (Muñiz, Galindo \& Garcia-López, 2003). En resumen, la RMB es un espacio aún fuertemente centralizado alrededor de las proximidades del municipio de Barcelona, pero una vez superado dicho ámbito, conforma un sistema urbano policéntrico, discontinuo y disperso.

La RMB está comunicada mediante una red de transporte radial, donde las principales concentraciones de población están conectadas con el centro de la metrópoli por diversas líneas ferroviarias (Renfe), carreteras y autopistas (A2, C32, C17). El crecimiento radial ha estado históricamente muy influenciado por condiciones topográficas que encauzaban la expansión de la metrópoli hacia los estrechos pasos entre cordilleras, los sistemas fluviales (los ríos Llobregat y Besós) y el corredor litoral hacia el norte. Más recientemente, se mejoraron las conexiones en el eje de costa sur (C32 sur) y se ha corregido el exceso de radialidad mediante conexiones transversales (AP7, B30, N340). Tanto estas condiciones infraestructurales como las topográficas han tenido una importante influencia en el patrón de urbanización (Miralles, 1997; Muñiz et al., 2003) (Mapa 1).

La división comarcal de Catalunya aprobada en 1936 se reinstauró en 1987 por el Gobierno de la Generalitat de Catalunya. Con algunas modificaciones respecto del formato original, en la actualidad Catalunya se divide en 41 comarcas, cuyo radio no suele exceder los $15 \mathrm{~km}$.

4 Terrassa, a pesar de no ser capital de comarca, tiene un peso histórico que la sitúa en el mismo nivel que Sabadell, los dos grandes núcleos "gemelos" de la comarca del Vallès Occidental. 
MAPA 1 División comarcal de Catalunya y ámbito espacial de la RMB (en gris oscuro)

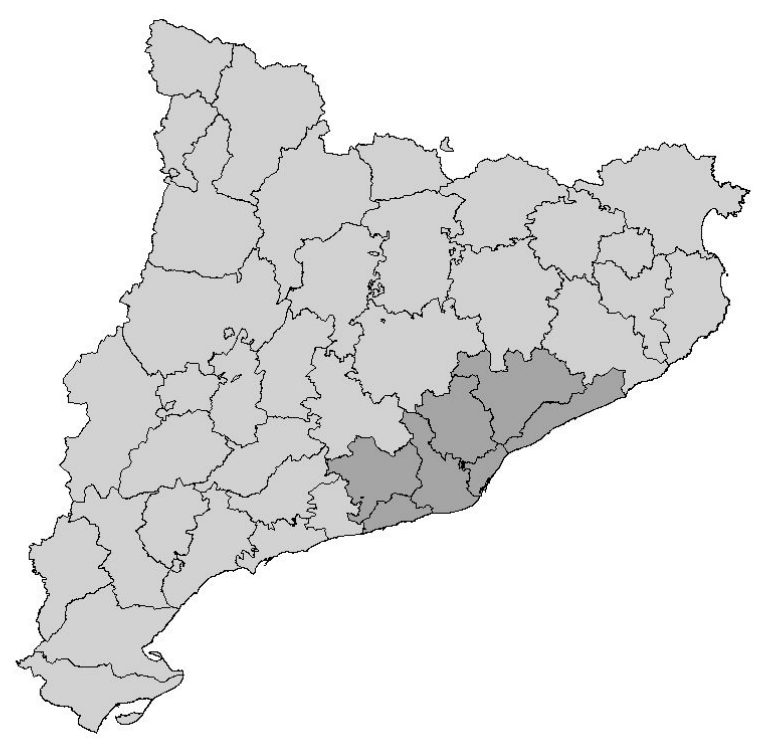

FUENTE ELABORACIÓN PROPIA.

\section{Dispersión espacial en la RMB. Estudios previos}

Son numerosos los trabajos que han abordado el problema de la dispersión urbana en la Región Metropolitana de Barcelona (Cuadro 1). Respecto de la ocupación del suelo, cabe citar la controversia entre los trabajos de Solans $(1998,2002)$ y Serratosa $(1998,1999)$, entre otros. ${ }^{5}$ La morfología de los asentamientos y su evolución han sido examinadas por el equipo de Antonio Font, de la Escuela Técnica Superior de Arquitectura de Valencia (ETSAV) (Font, Llop \& Vilanova, 1999; Font, Mas, Maristany, Carreras \& Valls, 2005) mediante planos detallados convenientemente actualizados, permitiendo con ello el registro pormenorizado del proceso de crecimiento metropolitano y su análisis. La expansión de la vivienda unifamiliar ha sido estudiada, entre otros, por Trilla (1997) y Muñoz (2005), y los procesos de suburbanización de la población, por Cabré y Mòdenes (1997) y Serra (1997). Otros aspectos analizados han sido el papel del planeamiento (Monclús, 1998a, 1998b) y los cambios en el modelo de transporte (Miralles, 1997). Son también interesantes los trabajos llevados a cabo por ecólogos como Rueda $(1998,2002)$, Folch (1998), y Mallarach y Comas (2005) sobre el impacto ambiental local de la ciudad 
dispersa. En cuanto a los estudios sobre estructura urbana, en Muñiz et al. (2003), Muñiz, Garcia-López y Galindo( 2008) y Garcia-López (2008, 2010) se investiga la relación entre descentralización de la población y densidad en un contexto policéntrico, y en Garcia-López y Muñiz (2010) y Garcia-López (2006) se lleva a cabo un estudio similar para el empleo. También diferentes trabajos han puesto de manifiesto la posibilidad de que los municipios dormitorio de la primera corona, urbanizados mediante polígonos de vivienda masiva durante las décadas de los sesenta y setenta, estén experimentando lo que se ha dado en llamar gothic urbanization (Short, Hanlon \& Vicini, 2007); esto es, un proceso prematuro de decadencia, debido a la corta edad del stock de viviendas, que los está convirtiendo en espacios de precariedad. Su localización y dotación de infraestructuras de transporte, y el perfil laboral de sus habitantes, han impedido que dichos municipios participen del dinamismo económico, tanto del centro principal como de los nuevos barrios suburbanos de baja densidad localizados en la periferia de la región (Ferrer, 2003; Muñiz \& Roig, 2001).

Por otro lado, la arquitectura de la dispersión en la RMB no ha sido demasiado diferente a la que se ha dado en otras ciudades españolas sometidas a presiones urbanísticas similares. Se trata, en general, de urbanizaciones que parecen clonadas: un espacio antropológicamente interesante en términos de no lugares, pues de forma similar a lo sucedido con los barrios dormitorio de la primera corona durante los años sesenta y setenta, tampoco en este caso parece posible dotarlo de algo parecido a la sensación de lugar. Estas "urbanalizaciones", siguiendo la terminología de Muñoz (2008), son el resultado apresurado de una sociedad menos arraigada a lo local, más globalizada en lo cultural y más preocupada por el bienestar que por participar de un proyecto social urbano compartido.

El caso de la Región Metropolitana de Barcelona es un buen ejemplo de cómo puede analizarse el fenómeno de la dispersión cuando no existe un acuerdo sobre sus rasgos fundamentales (Cuadro 1). El consumo de suelo, la baja densidad residencial y la fragmentación o discontinuidad son las tres dimensiones de la dispersión más citadas. Cabe destacar también que, excepto en contadas excepciones (Font et al., 1999, 2005), la dispersión del empleo ha estado ausente del debate, centrándose en el caso de la población o en el consumo de suelo en general.

La cuantificación de la dispersión en la RMB se ha limitado a las dimensiones descentralización y baja densidad. Se detecta, por tanto, la falta de estudios de naturaleza cuantitativa sobre las dimensiones desconcentración y discontinuidad, así como un intento de cuantificación del carácter aleatorio o falto de estructura de la dispersión. Este trabajo pretende, en la medida de lo posible, cubrir dicho vacío. Sin negar la importante contribución de los trabajos previos que han abordado el fenómeno de la dispersión en la RMB, algunos de los cuales presentan un rigor más allá de cualquier duda, el objetivo de este estudio es describir de forma homogénea, sistemática y lo más completa posible, el proceso de dispersión de la población y del empleo que se ha dado en la RMB entre 1986 y 2001. 
CUADRO 1 Elementos incorporados en la definición de dispersión aplicados sobre la RMB

\begin{tabular}{|c|c|c|c|c|c|}
\hline & 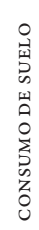 & 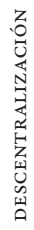 & 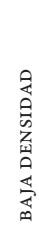 & 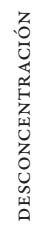 & 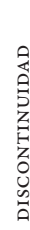 \\
\hline Carrera (2002) & & $\mathrm{X}$ & & $\mathrm{X}$ & \\
\hline Carreras (2002) & $\mathrm{X}$ & & & $\mathrm{X}$ & \\
\hline Solans (2002) & $\mathrm{X}$ & & & & \\
\hline Rueda (2002) & $\mathrm{X}$ & & $\mathrm{X}$ & & $\mathrm{X}$ \\
\hline Nel.lo $(1999,2002)$ & $\mathrm{X}$ & $\mathrm{X}$ & & $\mathrm{X}$ & \\
\hline Monclús (1998) & & $\mathrm{X}$ & & $\mathrm{X}$ & $\mathrm{X}$ \\
\hline Serratosa (1998) & $\mathrm{X}$ & & $\mathrm{X}$ & & $\mathrm{X}$ \\
\hline Muñoz $(2003,2005)$ & & & $\mathrm{X}$ & & \\
\hline Miralles (1997) & & $\mathrm{x}$ & $\mathrm{X}$ & & $\mathrm{X}$ \\
\hline Font et al. $(1999,2005)$ & $\mathrm{X}$ & $\mathrm{X}$ & $\mathrm{X}$ & & $\mathrm{X}$ \\
\hline Roca et al. (2004) & & & $\mathrm{X}$ & & $\mathrm{X}$ \\
\hline Paül y Tonts (2005) & & $\mathrm{x}$ & $\mathrm{X}$ & & $\mathrm{X}$ \\
\hline García y Riera (2003) & & & $\mathrm{X}$ & & \\
\hline Marull y Mallarach (2002) & $\mathrm{X}$ & & $\mathrm{X}$ & & $\mathrm{X}$ \\
\hline $\begin{array}{l}\text { Domene, Saurí y Pares } \\
\text { (2005) }\end{array}$ & $\mathrm{X}$ & & $\mathrm{X}$ & & \\
\hline Domene, Saurí (2006) & $\mathrm{X}$ & $\mathrm{x}$ & $\mathrm{X}$ & $\mathrm{X}$ & \\
\hline Durà-Guimerà (2003) & & & $\mathrm{X}$ & $\mathrm{X}$ & \\
\hline
\end{tabular}

FUENTE ELABORACIÓN PROPIA. 


\section{Los datos}

\section{La cuantificación de la dispersión}

El número de agentes económicos — población y empleo- proviene del padrón de población de 1986 y del censo de población de 2001. Los puestos de trabajo localizados se han obtenido a partir de los destinos de los desplazamientos obligados de la matriz de movilidad. Debido al carácter urbano del estudio, se ha considerado oportuno no incluir el empleo de los sectores agrícola, de extracción y de la energía.

La superficie de suelo proviene de los denominados Mapes d'Usos del Sól de Catalunya de los años 1987 y 2002. Estos mapas, elaborados por el Institut Cartogràfic de Catalunya (ICC), tienen su origen en datos multiespectrales del sensor Thematic Mapper del satélite Landsat-TM, con una escala de 1:25.000. La unidad mínima cartografiable es de 0,36 hectáreas y presenta una clasificación de veintiún usos, entre los cuales cuatro son urbanos (urbanizaciones, núcleos urbanos, zonas industriales y de servicios e infraestructuras). Aunque estos mapas son accesibles mediante internet en formato raster para el conjunto de Catalunya, la elaboración del mapa de la Región Metropolitana de Barcelona, así como el cómputo de la superficie que, a nivel municipal, se asocia a cada uno de los usos, se ha llevado a cabo mediante tecnología SIG. Es necesario destacar que, lamentablemente, no existe un mapa de usos del suelo compatible con los anteriores con posterioridad a 2002. Esto implica una severa limitación, ya que impide analizar el periodo más reciente. Sin embargo, todo parece indicar que lo acontecido entre 2001 y 2006 (año en que explota la burbuja inmobiliaria y frena en seco la fiebre urbanizadora) no ha hecho más que profundizar aquellas tendencias, que ya estaban presentes durante los quince años anteriores.

Parte de los indicadores propuestos pretende capturar el papel estructurador de las infraestructuras viarias. La explotación del Institut Cartogràfic de Catalunya permite obtener la distancia perpendicular para el año 1997 de cada municipio respecto de la infraestructura viaria principal. La distancia temporal al acceso más cercano se ha obtenido del Departament de Política Territorial i Obres Públiques (DPTOP) de la Generalitat de Catalunya. Esta institución encargó a la consultora Mcrit, S.L. el programa SIMCAT, un software SIG para realizar simulaciones sobre la infraestructura viaria que incorpora las infraestructuras cada cinco años, desde 1986 a 2001.

Es necesario mencionar que no todos los 164 municipios que constituían la RMB en 2001 existían en 1986. En 1995 y 1999 se crearon dos municipios nuevos, Badia del Vallès y La Palma de Cervelló, a partir de su escisión de Cerdanyola del Vallès y Cervelló, respectivamente. Para mantener el número de observaciones constante se ha decidido trabajar con 162 municipios. La información de los municipios escindidos se ha incorporado a los municipios de origen.

\section{Los indicadores}

Descentralización. El indicador utilizado para caracterizar la descentralización de la población y del empleo es la distancia media ponderada al CBD (central business dis- 
trict, centro o distrito financiero) (Galster et al., 2001; Glaeser \& Kahn, 2004; Malpezzi \& Guo, 2001). Otros indicadores alternativos son la densidad teórica central (Malpezzi \& Guo, 2001; Ewing, Pendall \& Chen, 2002) y el gradiente de densidad estimados a partir de una función de densidad exponencial (Bertaud \& Malpezzi, 1999; Torrens \& Alberti, 2000; Malpezzi \&Guo, 2001; Couch et al., 2005), el porcentaje de población y/o empleo en el centro (Galster et al., 2001; Wassmer, 2000), o el porcentaje de población y/o empleo a menos de tres millas del centro (Glaeser \& Kahn, 2004). Debe recordarse que en este trabajo la descentralización se considera una condición necesaria pero no suficiente para que se dé dispersión.

Densidad. Al igual que en otros trabajos, el indicador de intensidad de uso del suelo es la densidad neta (Malpezzi \& Guo, 2001; Galster et al., 2001; Wassmer \& Edwards, 2005; Wolman et al., 2005; Glaeser \& Kahn, 2004; Song \& Knaap, 2004; Kahn, 2006). Otro indicador de densidad utilizado en ocasiones es el porcentaje de población y/o empleo localizado en condiciones de baja densidad, entendiendo por baja densidad la que esté por debajo de un determinado umbral (Ewing et al., 2002).

Concentración. Se mide mediante el índice de Gini (Tsai, 2005). Otros indicadores similares utilizados en diferentes trabajos son el porcentaje de población y/o empleo que vive en zonas muy densas, la desviación típica de la densidad, el índice de disimilitud de Massey y Denton (Galster et al., 2001; Wolman et al., 2005), o los índices de Theil (Tsai, 2005) y Geary (Malpezzi \& Guo, 2001).

Discontinuidad. El grado de discontinuidad se aproxima a partir del número de bolsas urbanas (islas de continuidad edificada) y un indicador especialmente construido donde el número de bolsas se pondera por la población del municipio. Este indicador también puede calcularse a escala metropolitana.

Desestructuración o desvertebración del territorio. Se estima contrastando si la distancia ponderada a la infraestructura de transporte y a los accesos ha aumentado. Adicionalmente se estima la concentración de población y empleo en subcentros en 1986 y 2001 . Si su peso descendiera, indicaría un menor policentrismo y, por tanto, una mayor dispersión.

\section{Consumo de suelo y descentralización}

Entre 1986 y 2001, la población de la Región Metropolitana de Barcelona pasó de 4.229.422 a 4.390.413. La tasa de crecimiento fue del 3,8\%. Durante el mismo periodo, el número de puestos de trabajo aumentó de forma significativa, pasando de 1.063 .283 a 1.822 .819 , lo cual representa una tasa de crecimiento del $71,4 \%$. Por último, la superficie urbanizada se incrementó en 25.503 hectáreas. Con una tasa de crecimiento del 60\%, el suelo urbanizado pasó de 42.496 a 67.999 hectáreas (Cuadro 2). Los datos indican que el ritmo de consumo de suelo ha sido considerablemente superior al de la población, pero no al del empleo. 
Entre 1986 y 2001, la distancia media ponderada de la población respecto del CBD ha pasado de $10,2 \mathrm{~km}$ a $12,1 \mathrm{~km}$; y la del empleo, de $9,8 \mathrm{~km}$ a $11,1 \mathrm{~km}$. Se detecta, por tanto, una clara tendencia hacia la descentralización ${ }^{6}$ (Cuadro 3).

\section{Dispersión y forma urbana: desconcentración, baja densidad y discontinuidad}

\section{a) Desconcentración}

La caída en el valor del índice de $\mathrm{Gini}^{7}$ aplicado al volumen de población y de empleo refleja la desconcentración de los agentes en el territorio. La diferencia entre los municipios más y menos poblados, así como aquellos que cuentan con más y menos empleo, se ha reducido. Por lo tanto, la población y la actividad económica han tendido a distribuirse de forma más equilibrada en el ámbito metropolitano (Cuadro 3).

CUADRo 2 Consumo de suelo, población y empleo en la RMB, 1987-2002: superficies

\begin{tabular}{|c|c|c|c|}
\hline & 1987 & 2002 & $1987-2002$ \\
\hline \multirow{2}{*}{ Suelo urbanizado (ha) } & 42.496 & 67.999 & 25.503 \\
\hline & $(13,1 \%)$ & $(20,9 \%)$ & $(60 \%)$ \\
\hline \multirow{2}{*}{ Suelo en resto de usos (ha) } & 281.778 & 256.284 & -25.493 \\
\hline & $(86,8 \%)$ & $(79 \%)$ & $(-9 \%)$ \\
\hline Población & 4.229 .527 & 4.390 .413 & $\begin{array}{r}160.886 \\
(3.8 \%)\end{array}$ \\
\hline \multirow{2}{*}{ Empleo } & \multirow{2}{*}{1.063 .283} & \multirow{2}{*}{1.822 .819} & 759.536 \\
\hline & & & $(71,4 \%)$ \\
\hline
\end{tabular}

FUENTE ELABORACión PROPIA CON DATOS DE SUELO PROCEDENTES De los MAPES D'USOS DEL Sól DE CATALUNYA (INSTITUT CARTOGRÀFIC DE CATALUNYA, ICC) Y DATOS DE POBLACIÓN Y DE PUESTOS DE TRABAJO LOCALIZADOS procedentes del Padrón Municipal de Habitantes de 1986 y el Censo de Población de 2001 (Instituto NACIONAL DE ESTADÍSTICA).

6 La distancia media ponderada ha sido calculada como $D C B D_{i}=\sum_{i}\left(d_{i, C B D} s_{i, t}\right)$ donde $d_{i, C B D}$ es la distancia que separa el municipio $i$ del CBD y $S_{i, t}$ es la proporción de agentes (población/empleo) que acumula el municipio respecto el total de la RMB.

7 El índice de Gini municipal permite evaluar el grado de concentración municipal, es decir, hasta qué punto los agentes se han concentrado en unas pocas observaciones (municipios): $G M_{t}=1-\sum\left(s_{i, t}+s_{i-1, t}\right)(1 / n)$. En la curva de Lorenz asociada se grafican los valores acumulativos de $1 / n$ (porcentajes municipales) en el eje de ordenadas respecto al número acumulado de municipios en el de abscisas (cada intervalo con la misma amplitud, $1 / n$ ). 
CUADRO 3 Indicadores de dispersión en la RMB, 1986-2001

\begin{tabular}{|c|c|c|c|c|}
\hline & \multicolumn{2}{|c|}{1986} & \multicolumn{2}{|c|}{2001} \\
\hline & POBLACIÓN & EMPLEO & POBLACIÓN & EMPLEO \\
\hline Descentralización & & & & \\
\hline Distancia media ponderada al CBD $(\mathrm{km})$ & 10,2 & 9,8 & 12,1 & 11,1 \\
\hline \multicolumn{5}{|l|}{ Desconcentración } \\
\hline Índice de Gini & 0,85 & 0,85 & 0,79 & 0,81 \\
\hline \multicolumn{5}{|l|}{ Baja densidad } \\
\hline Densidad neta (agentes por hectárea) & 148,2 & 39,4 & 96,2 & 67,2 \\
\hline \multicolumn{5}{|l|}{ Discontinuidad } \\
\hline \multicolumn{5}{|l|}{ Número de bolsas } \\
\hline \multirow{2}{*}{$\begin{array}{l}\text { Bolsas / Suelo urbanizado (sin } \\
\text { infraestructuras) }\end{array}$} & \multicolumn{2}{|l|}{3.105} & \multicolumn{2}{|l|}{2.468} \\
\hline & 0,059 & & 0,043 & \\
\hline \multicolumn{5}{|l|}{ Desestructuración (I) } \\
\hline \multicolumn{5}{|l|}{ Crecimiento lineal } \\
\hline Distancia perpendicular infraestructura $(\mathrm{km})$ & 1,1 & 1,2 & 1,1 & 1,1 \\
\hline Distancia acceso viario $(\mathrm{km})$ & 3,6 & 3,7 & 2,6 & 2,6 \\
\hline \multicolumn{5}{|l|}{ Desestructuración (II) } \\
\hline \multicolumn{5}{|l|}{ Policentrismo } \\
\hline Número de subcentros & & 6 & & 9 \\
\hline$\%$ agentes en subcentros (año $t$ ) & & $14,8 \%$ & & $18,0 \%$ \\
\hline
\end{tabular}

FUENTE ELABORACión PROPIA CON DATOS DE SUELO PROCEDENTES DE LOS MAPES D'USOS DEL SÓL DE CATALUNYA (INSTITUT CARTOGRÀFIC DE CATALUNYA, ICC) y DATOS DE POBlaCióN Y DE PUESTOS DE TRABAJO LOCALIZADOS procedentes del Padrón Municipal de Habitantes de 1986 y el Censo de Población de 2001 (Instituto Nacional de Estadística). Distancias Calculadas mediante SIG.

\section{b) Baja densidad}

La densidad neta de población ha caído de forma significativa, pasando de 148,2 a 96,2 habitantes por hectárea (Cuadro 3). La caída de la densidad de población ha sido posible gracias a importantes cambios en la edificación, localización y uso de 
las viviendas. Los datos del Censo de Viviendas indican que el número de viviendas totales de la RMB ha pasado de 1.729 .717 a 2.063.975 entre 1991 y 2001, un incremento neto de 334.258 viviendas, que supone una tasa de crecimiento del $20 \%{ }^{8}$ (Cuadro 4). Atendiendo a los datos anteriores, cabría esperar una cierta correspondencia entre la caída de la densidad y un mayor peso de las viviendas unifamiliares. Sin embargo, los datos lo desmienten. Con una tasa de crecimiento de tan solo el 9,3\%, el número de viviendas unifamiliares ha pasado de 282.787 en 1991 a 309.138 en 2001 , pero su peso respecto del total de viviendas se ha reducido, cayendo del 16,3\% al 14,9\% (Cuadro 4).

\section{MAPA 2 Densidad neta de población en la RMB, 2001}

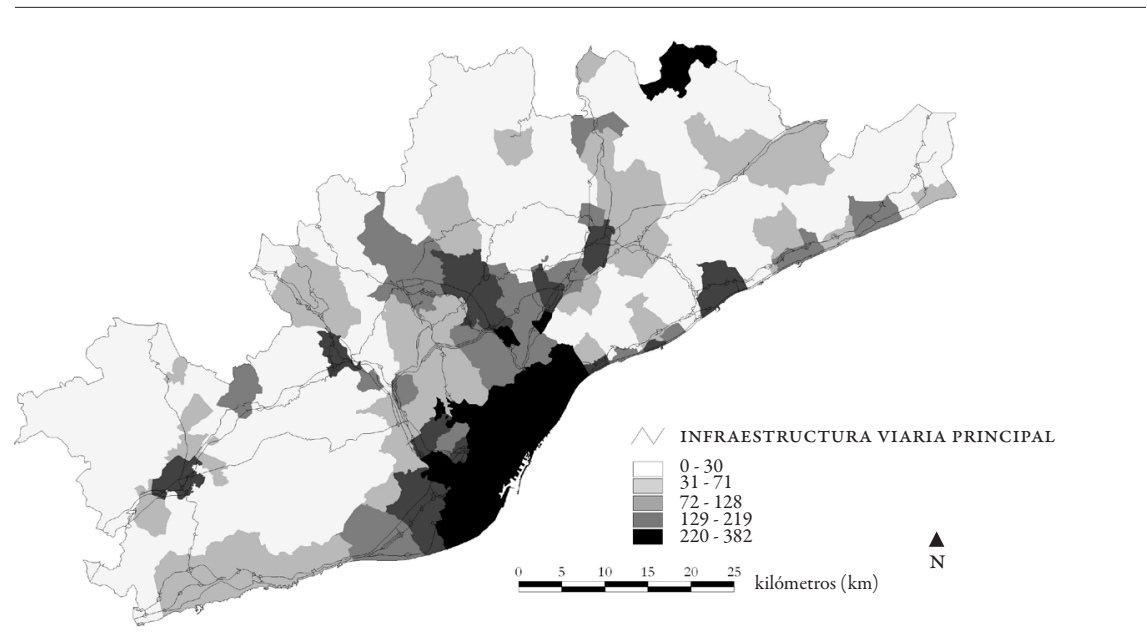

FUENTE ELABORACIÓN PROPIA CON DATOS DE POBLACIÓN PROCEDENTES DEL CENSO De POBLACIÓN DE 2001 (INSTITUTO NACIONAL de ESTADística).

Estos resultados esconden, sin embargo, un fenómeno de capital importancia: el paso de segundas a primeras residencias. El porcentaje de personas que vive habitualmente en una vivienda unifamiliar no ha crecido 9,3\%, sino 26,5\% (Cuadro 4). Ha sido de tal magnitud el cambio de usos que, pese a haberse dado un fuerte incremento en el número de viviendas unifamiliares para ser utilizadas como segundas residencias, el saldo del número de viviendas unifamiliares no principales entre 1991 y 2001 presenta un signo negativo. En 2001 había 21.484 viviendas unifamiliares no principales menos que en 1991.

En el caso del empleo, su densidad neta ha aumentado de 54 a 67 puestos de trabajo por hectárea (Cuadro 3). Este resultado se debe a que el intenso crecimiento del empleo se ha localizado en un espacio que en gran medida ya estaba disponible en 1986. El principal cambio ha consistido en utilizarlo de forma más intensiva.

8 El censo de viviendas se lleva a cabo cada diez años, por lo que no se dispone de datos para el año 1986. Se ha constatado que la evolución desde 1981 no se diferencia significativamente de la que se da a partir de 1991, por lo que se ha decidido no incluir esta información. 
CUADRO 4 Viviendas en la RMB, 1991-2001: unidades, peso y crecimiento

\begin{tabular}{|c|c|c|c|}
\hline & 1991 & 2001 & $1991-2001$ \\
\hline Todas las viviendas & 1.729 .719 & 2.063 .975 & $\begin{array}{c}334.256 \\
(19,32 \%)\end{array}$ \\
\hline Todas las unifamiliares & $\begin{array}{c}282.787 \\
(16,3 \%)^{*}\end{array}$ & $\begin{array}{c}309.138 \\
(14,9 \%)^{*}\end{array}$ & $\begin{array}{c}26.351 \\
(9,3 \%)\end{array}$ \\
\hline Viviendas principales & $\begin{array}{l}1.365 .808 \\
(78,9 \%)^{*}\end{array}$ & $\begin{array}{c}1.607 .347 \\
(77,8 \%)^{*}\end{array}$ & $\begin{array}{c}241.539 \\
(17,6 \%)\end{array}$ \\
\hline Unifamiliares principales & $\begin{array}{l}173.281 \\
(10 \%)^{*} \\
(12,6 \%)^{* *}\end{array}$ & $\begin{array}{c}219.337 \\
(10,6 \%)^{*} \\
(13,6 \%)^{* *}\end{array}$ & $\begin{array}{c}46.056 \\
(26,5 \%)\end{array}$ \\
\hline Viviendas no principales & $\begin{array}{l}363.911 \\
(21 \%)^{*}\end{array}$ & $\begin{array}{c}441.692 \\
(21,4 \%)^{*}\end{array}$ & $\begin{array}{c}77.781 \\
(21,3 \%)\end{array}$ \\
\hline $\begin{array}{l}\text { Unifamiliares no } \\
\text { principales }\end{array}$ & $\begin{array}{c}109.506 \\
(6,3 \%)^{*} \\
(30 \%)^{* * *}\end{array}$ & $\begin{array}{c}88.022 \\
(4,2 \%)^{*} \\
(19,9 \%)^{* * *}\end{array}$ & $\begin{array}{l}-21.484 \\
(-19,6 \%)\end{array}$ \\
\hline
\end{tabular}

* Porcentaje ReSpecto total viviendas. ${ }^{* *}$ Porcentaje ReSpecto viviendas PrinCipales. ${ }^{* * *}$ Porcentaje ResPECTO VIVIENDAS NO PRINCIPALES.

FUENTE Elaboración propia con datos de ViViendas procedentes de los Censos de ViViendas de 1991 Y 2001 (INSTITUTO NACIONAL DE ESTADÍSTICA).

\section{MAPA 3 Densidad neta de empleo en la RMB, 2001}

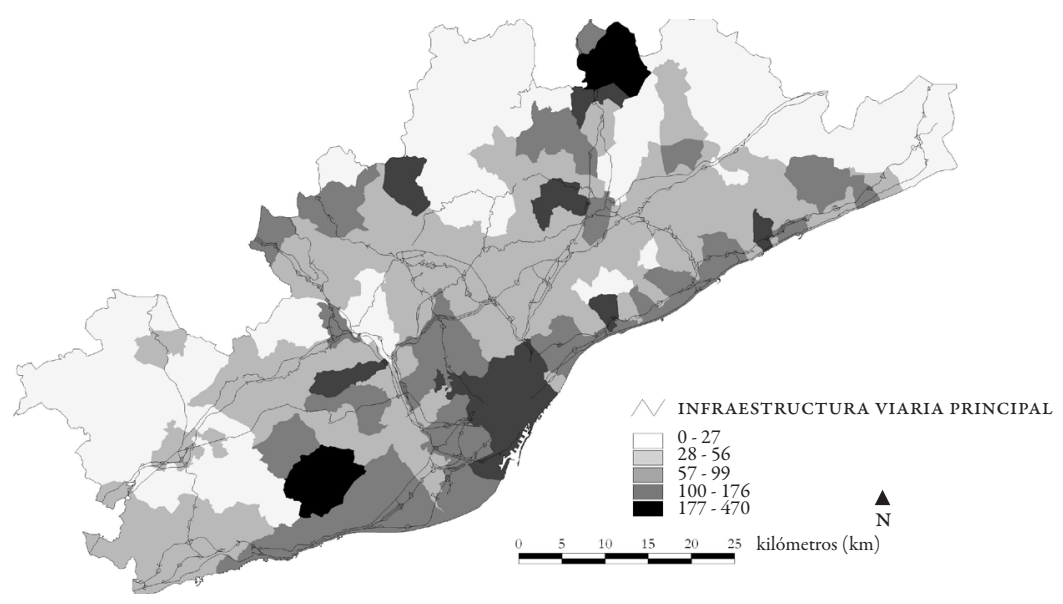

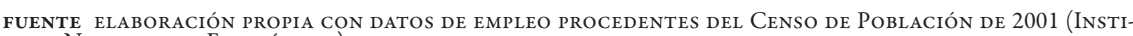
TUto Nacional de Estadística). 


\section{c) Discontinuidad}

Para analizar la evolución de la discontinuidad, se parte del suelo urbano con independencia del uso que se le dé (residencial o productivo), conformado en bolsas. Cada bolsa se corresponde con un espacio edificado continuo. Según el Mapa d' Usos del Sòl de la Regió Metropolitana de Barcelona, el número de bolsas que había en 1987 (3.105), supera al que había en 2002 (2.468). Parece, por tanto, que entre 1987 y 2002 el territorio no ha tendido a dispersarse de forma discontinua, sino todo lo contrario. Existen muchos casos donde bolsas que en 1987 estaban a una corta distancia se han anexionado formando una sola. Este resultado no desmiente el carácter fragmentado y discontinuo de la RMB. Sugiere, en cambio, que la discontinuidad no es un fenómeno reciente. La fragmentación que hoy puede observarse ya estaba presente en 1987 y, probablemente, con bastante anterioridad, a juzgar por los resultados obtenidos por Font et al. (2005) al comparar los tejidos discontinuos de 2000 con los de 1977. A grandes rasgos, se detecta un aumento en el número de bolsas en los municipios cercanos a Granollers, en la costa del Maresme y, en general, en los márgenes interiores de la RMB. En el otro extremo, los municipios que en mayor medida han consolidado bolsas urbanas están localizados en el continuo de Barcelona, en el Baix Llobregat, en el corredor de Caldes y en las proximidades de Vilanova.

Una forma de capturar la evolución de la discontinuidad para el conjunto de la RMB teniendo en cuenta no solo el número de bolsas, sino también el espacio que ocupan, consiste en calcular el ratio entre el número de bolsas y el suelo urbanizado total (excepto las infraestructuras). El aumento del número de bolsas, permaneciendo estable el suelo urbanizado, implica una mayor discontinuidad. Si, por el contrario, el número de bolsas permanece estable, pero aumenta el suelo urbanizado, el indicador caería de valor, es decir, indicaría una menor discontinuidad, ya que con un número igual de bolsas ha sido posible urbanizar más territorio. Para el caso de la RMB en su conjunto, dicho ratio ha pasado de 0,059 en 1987 a 0,043 en 2002.

MAPA 4 Variación municipal en el número de bolsas de suelo urbano, 1987- 2002

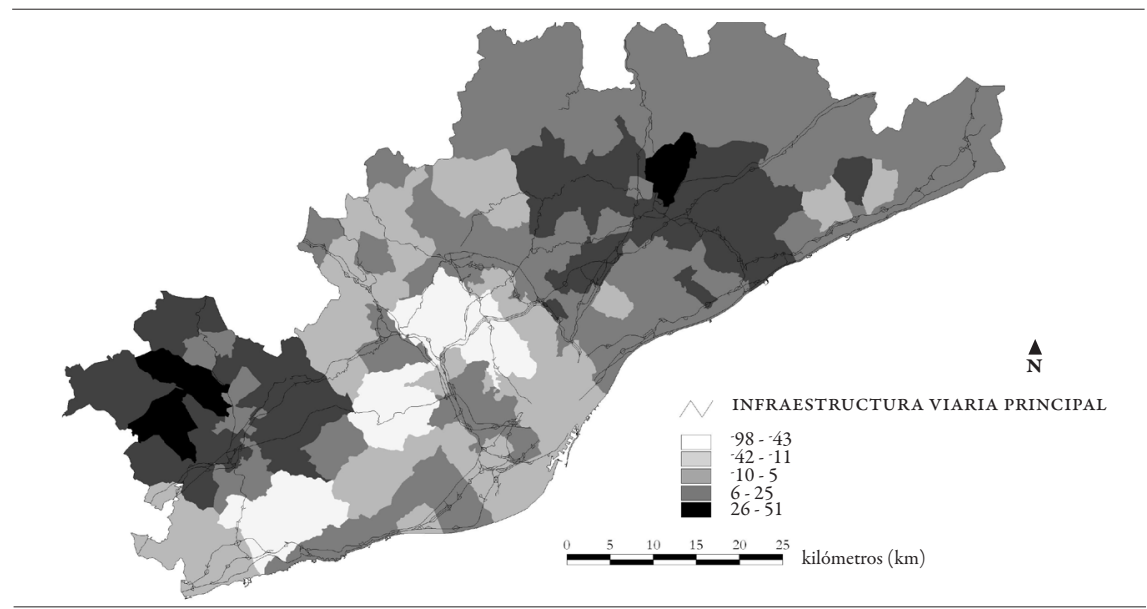

FUENTE ELABORACión PRopia CON DATOS DE SUELO PRocedentes de los MAPES D'USOS DEL SÓL DE CATALUNYA DE 1987 Y 2002 (INSTITUT CARTOGRÀFIC DE CATAlunya, ICC). 
Dispersión y estructura urbana: Proximidad a la infraestructura viaria y el peso de los subcentros

Tomando como referencia el mapa de infraestructuras viarias de 1997, la distancia media perpendicular — ponderada por el peso poblacional- de cada municipio al eje viario más próximo se ha mantenido invariable entre 1986 y 2001, con un valor de $1,1 \mathrm{~km}$. En el caso del empleo, ha pasado de $1,2 \mathrm{~km}$ a $1,1 \mathrm{~km}$, lo que supone un ligero acercamiento. En cuanto a la distancia media ponderada respecto del acceso viario más próximo, ha caído de $3,6 \mathrm{~km}$ a $2,68 \mathrm{~km}$ para la población, y de $3,7 \mathrm{~km}$ a $2,6 \mathrm{~km}$ para el empleo (Cuadro 3). El aumento de la población y del empleo ha sido, por tanto, más intenso cerca de los accesos a las principales infraestructuras viarias, debido tanto a un acercamiento de los agentes a los accesos como a mejoras en estos últimos, que ha permitido acercarlos a los agentes.

MAPA 5 Distancia $(\mathrm{km})$ perpendicular a la infraestructura viaria en la RMB, 1997

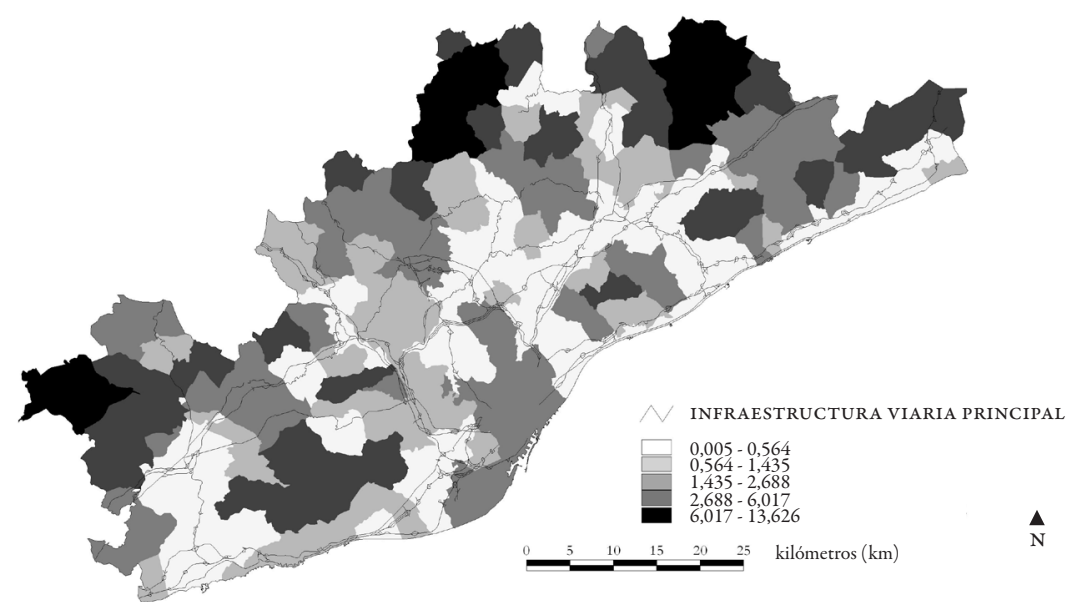

FUENTE ELABORACIÓN PROPIA CON DATOS SOBRE INFRAESTRUCTURA VIARIA PRINCIPAL (INSTITUT CARTOGRÀFIC de Catalunya, ICC).

Aplicando los umbrales estadísticos al porcentaje de empleo y a las condiciones de densidad' (una densidad de población o empleo por encima de la densidad media de la región y un porcentaje municipal de población o empleo superior al $1 \%$ del total) como criterio para la identificación de subcentros de empleo, el número de municipios que cumplía con ambas condiciones y que, por tanto, podemos catalogar como subcentros en 1986 y en 2001, aumenta de 6 a $9^{10}$ (Cuadro 3).

9 Véase Garcia-López y Muñiz (2007) y Muñiz et al. (2008).

10 En 1986, los subcentros de empleo eran Granollers, Mataró, Rubí, Sabadell, Terrassa y Vilanova i la Geltrú. En 2001 se incorporaron a la lista los municipios de Martorell, Cerdanyola del Vallès y Sant Cugat del Vallès. 
MAPA 6 Distancia $(\mathrm{km})$ al acceso más próximo de la infraestructura viaria principal, 2001

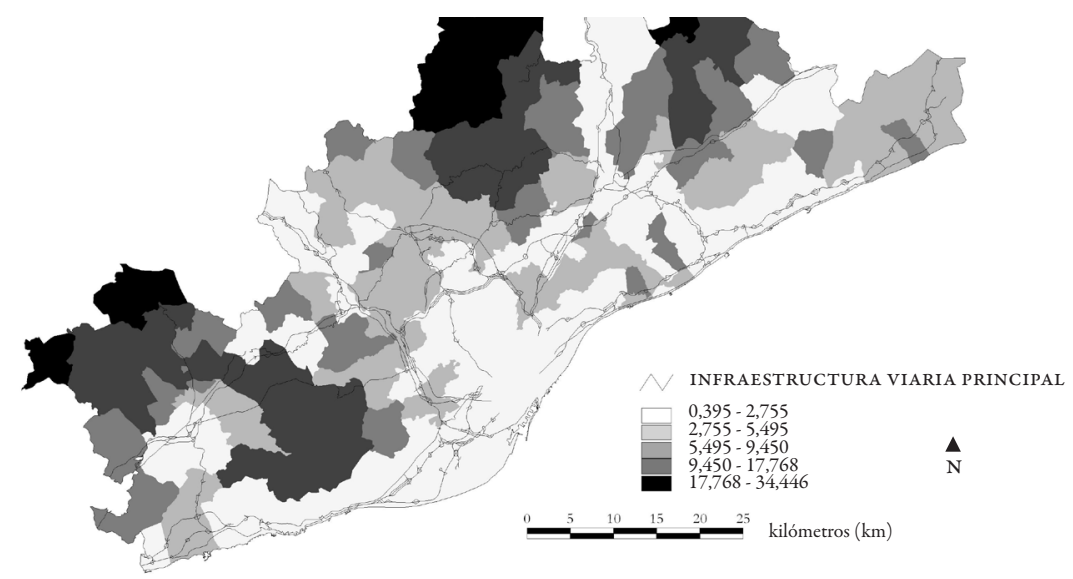

FUENTE ELABORACIÓN PROPIA CON DATOS SOBRE INFRAESTRUCTURA VIARIA PRINCIPAL (INSTITUT CARTOGRÀFIC de Catalunya, ICC).

MAPA 7 Subcentros de empleo en la RMB, 1986-2001

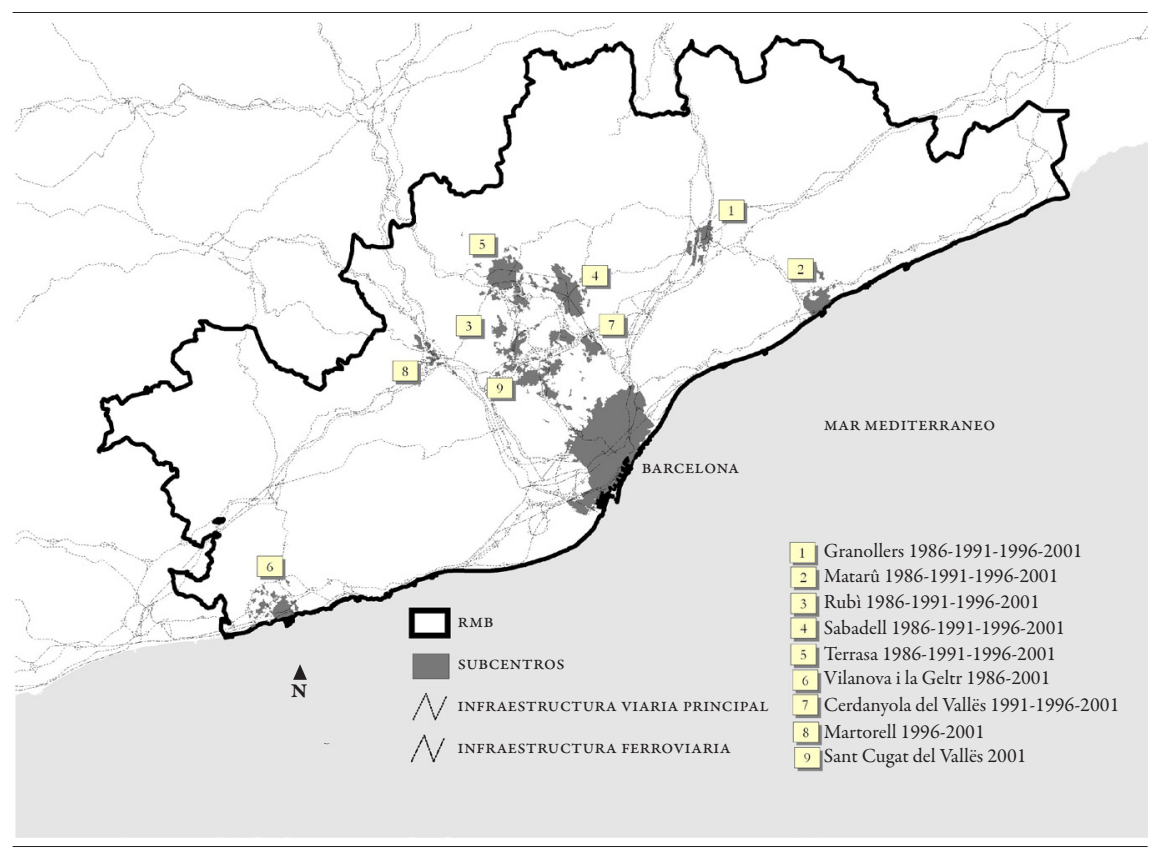

fuente elaboración propia con datos de empleo procedentes del Censo de Población de 2001 (Instituto NaCional de Estadística). 
GRÁFICO 1 Identificación de los subcentros de empleo en la RMB.

Coincidencia con los picos de una función de densidad de empleo LWR

$1986-2001$
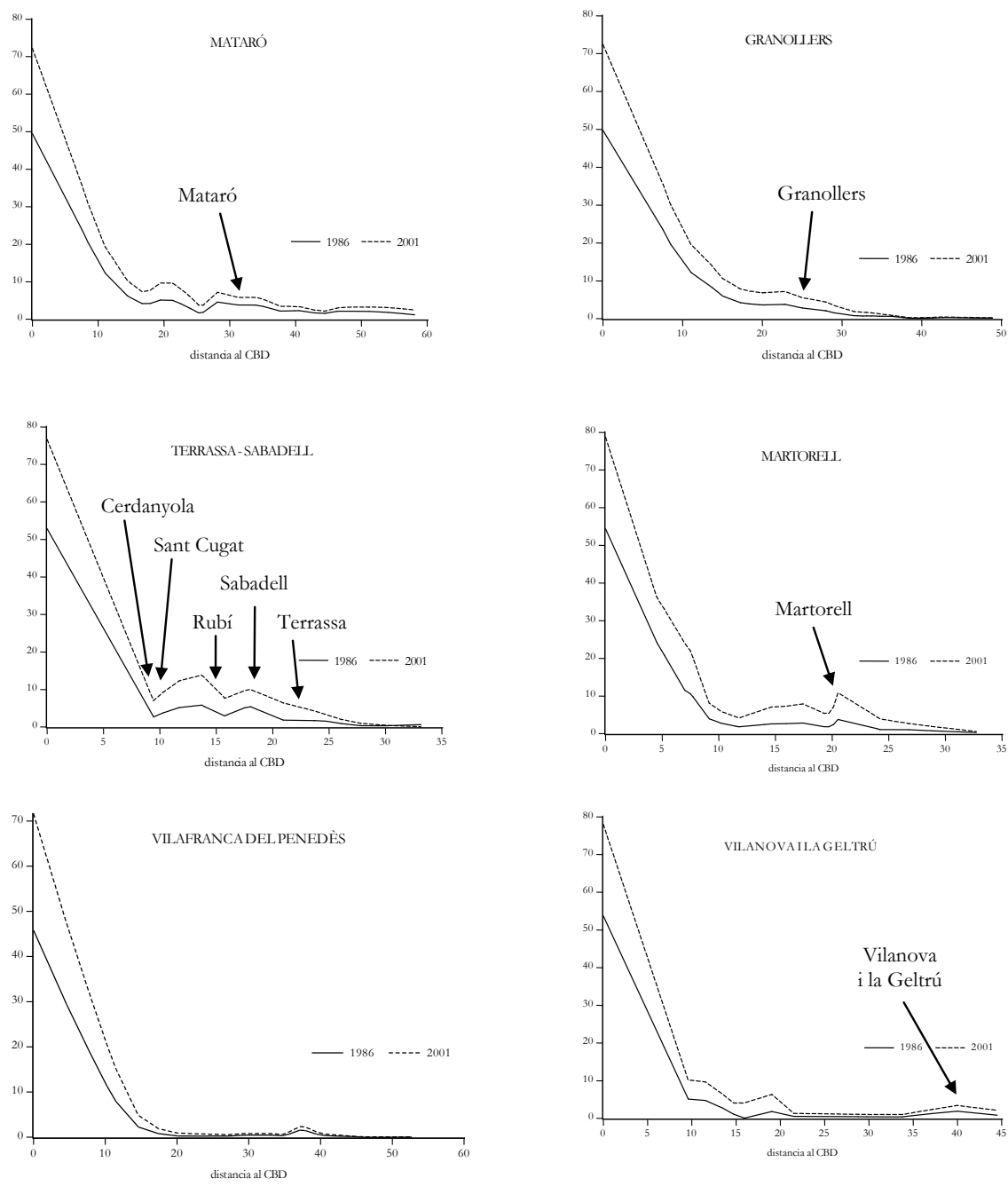

FUENTE ELABORACIÓN PROPIA.

A pesar de que la población concentrada en el CBD ha caído sensiblemente, pasando de representar el 64,6\% al 56,1\% del total de la RMB (Cuadro 5), el peso de la población localizada en subcentros ha aumentado, tanto si se incorporan en el cálculo los nuevos subcentros identificados en 2001 (9) como si solo se tienen 
en cuenta los subcentros identificados en 1986 (6). Ahora bien, al considerar solo los subcentros identificados en 1986 como referencia, el crecimiento de la población ha sido más intenso en el grupo de "otros municipios". Por otro lado, tomando como referencia los seis subcentros identificados en 1986 y los nueve en 2001, el porcentaje de puestos de trabajo localizados en subcentros pasa de representar el 14,8\% a alcanzar el 18\% (Cuadro 6). La tasa de crecimiento del empleo en los subcentros $(108,6 \%)$ supera ligeramente a la que se da de forma más dispersa en el grupo de "otros municipios" (106\%). No sucede lo mismo cuando se consideran tan solo los subcentros identificados en 1986. En tal caso, el crecimiento del empleo es mayor en "otros municipios" que en los subcentros, lo cual indica una cierta saturación de los subcentros tradicionales y un fuerte dinamismo de los emergentes.

CUADRO 5 Importancia y crecimiento de la población por ámbitos espaciales, 1986-2001

\begin{tabular}{|c|c|c|c|c|}
\hline & & 1986 & 2001 & $1986-2001$ \\
\hline \multirow{3}{*}{$\begin{array}{l}\text { Subcentros } \\
\text { año } t\end{array}$} & CBD & $\begin{array}{l}2.729 .955 \\
(64,6 \%)\end{array}$ & $\begin{array}{l}2.465 .234 \\
(56,1 \%)\end{array}$ & $\begin{array}{l}-264.721 \\
(-9,7 \%)\end{array}$ \\
\hline & Subcentros & $\begin{array}{c}639.176 \\
(15,1 \%)\end{array}$ & $\begin{array}{c}853.850 \\
(19,5 \%)\end{array}$ & $\begin{array}{c}214.674 \\
(33,6 \%)\end{array}$ \\
\hline & Otros municipios & $\begin{array}{l}860.396 \\
(20,3 \%)\end{array}$ & $\begin{array}{l}1.071 .329 \\
(24,4 \%)\end{array}$ & $\begin{array}{c}210.933 \\
(24,5 \%)\end{array}$ \\
\hline \multirow{3}{*}{$\begin{array}{l}\text { Subcentros } \\
1986\end{array}$} & CBD & $\begin{array}{l}2.729 .955 \\
(64,5 \%)\end{array}$ & $\begin{array}{l}2.465 .234 \\
(56,1 \%)\end{array}$ & $\begin{array}{l}-264.721 \\
(-9,7 \%)\end{array}$ \\
\hline & Subcentros & $\begin{array}{c}639.176 \\
(15,1 \%)\end{array}$ & $\begin{array}{c}706.746 \\
(16,1 \%)\end{array}$ & $\begin{array}{c}67.570 \\
(10,6 \%)\end{array}$ \\
\hline & Otros municipios & $\begin{array}{l}860.396 \\
(20,3 \%)\end{array}$ & $\begin{array}{l}1.218 .433 \\
(27,8 \%)\end{array}$ & $\begin{array}{c}358.037 \\
(41,6 \%)\end{array}$ \\
\hline
\end{tabular}

FUENTE ELABORACIÓN PROPIA CON DATOS DE POBLACIÓN PROCEDENTES DEL PADRóN MUNICIPAL DE HABITANtes de 1986 y el Censo de Población de 2001 (Instituto Nacional de Estadística).

Este modelo de descentralización concentrada del empleo ha permitido organizar la dispersión residencial alrededor de los subcentros. Se trata, por tanto, de un proceso de dispersión fuertemente articulado a partir de una estructura básica conformada por CBD, subcentros y ejes de transporte. El resultado final es fruto más de dinámicas de mercado que de la existencia de una planificación urbana coherente a escala regional, aunque es cierto que la política de dotación de infraestructuras 
viarias y el papel de la administración pública en la promoción de suelo destinado a industrias y servicios han servido a su fin de forma coherente (Ferrer, 2003; Vecslir, 2007). Sin embargo, hubiera sido deseable la existencia de un urbanismo de escala superior a la municipal capaz de coordinar las políticas de suelo, vivienda e infraestructuras de transporte, ya que esto hubiera permitido dimensionar mejor los barrios suburbanos, aumentar su densidad y mejorar su acceso al transporte público. Por otro lado, este tipo de planeamiento hubiera permitido frenar el proceso de decadencia de los barrios dormitorio de la primera corona y los procesos de competencia entre los municipios más periféricos, que ha llevado a una promoción excesiva de nuevos espacios residenciales (Nel.lo, 2002; Font, 2007).

CUADRO 6 Importancia y crecimiento del empleo por ámbitos espaciales 19862001

\begin{tabular}{|c|c|c|c|c|}
\hline & & 1986 & 2001 & $1986-2001$ \\
\hline \multirow{3}{*}{$\begin{array}{l}\text { Subcentros } \\
\text { año } t\end{array}$} & CBD & $\begin{array}{c}689.385 \\
(64,8 \%)\end{array}$ & $\begin{array}{l}1.048 .400 \\
(57,5 \%)\end{array}$ & $\begin{array}{c}359.015 \\
(52,1 \%)\end{array}$ \\
\hline & Subcentros & $\begin{array}{c}157.063 \\
(14,8 \%)\end{array}$ & $\begin{array}{c}327.698 \\
(18,0 \%)\end{array}$ & $\begin{array}{c}170.365 \\
(108,6 \%)\end{array}$ \\
\hline & Otros municipios & $\begin{array}{l}216.835 \\
(20,4 \%)\end{array}$ & $\begin{array}{c}446.721 \\
(24,5 \%)\end{array}$ & $\begin{array}{c}229.886 \\
(106,0 \%)\end{array}$ \\
\hline \multirow{3}{*}{$\begin{array}{l}\text { Subcentros } \\
1986\end{array}$} & CBD & $\begin{array}{c}689.385 \\
(64,8 \%)\end{array}$ & $\begin{array}{l}1.048 .400 \\
(57,5 \%)\end{array}$ & $\begin{array}{c}359.015 \\
(52,1 \%)\end{array}$ \\
\hline & Subcentros & $\begin{array}{c}157.063 \\
(14,8 \%)\end{array}$ & $\begin{array}{c}255.898 \\
(14,0 \%)\end{array}$ & $\begin{array}{c}98.835 \\
(62,9 \%)\end{array}$ \\
\hline & Otros municipios & $\begin{array}{c}216.835 \\
(20,4 \%)\end{array}$ & $\begin{array}{l}518.521 \\
(28,4 \%)\end{array}$ & $\begin{array}{c}301.686 \\
(139,1 \%)\end{array}$ \\
\hline
\end{tabular}

FUENTE ELABORACIÓN PROPIA CON DATOS DE EMPLEO PROCEDENTES DEL PADRón MUNICIPAL DE HABITANTES DE 1986 y el Censo de Población de 2001 (Instituto Nacional de Estadística).

\section{Resumen y conclusiones}

Los resultados obtenidos indican que, tanto en el caso de la población como en el del empleo, durante los quince años que abarca el estudio se ha dado un proceso de descentralización y desconcentración. Los agentes han tendido a localizarse más lejos del CBD, ocupando el territorio de forma más homogénea. La densidad de población ha caído, es decir, el ritmo de ocupación de suelo para usos residenciales 
ha sido superior al crecimiento de la población. Sin embargo, debe destacarse que el incremento de la vivienda unifamiliar ha sido menor que el de la plurifamiliar. En contraste con el caso de la población, la densidad de empleo ha aumentado. El importante crecimiento de puestos de trabajo se ha ubicado preferentemente en un espacio que ya estaba disponible en 1986, pero que probablemente se utilizaba por debajo de su potencial. Adicionalmente, la discontinuidad del territorio ha sido corregida al juntarse bolsas (infill) que en 1986 estaban separadas. Un resultado especialmente interesante es la constatación de que el crecimiento de la RMB no se está dando bajo un modelo caótico, sino crecientemente estructurado a partir de los centros y accesos a las infraestructuras de transporte. Si asimilamos dispersión con crecimiento aleatorio, la RMB no se ha dispersado, sino que se ha extendido siguiendo una estructura cada vez más definida. OEURE

\section{Referencias bibliográficas}

Allen, A. (2003). Environmental planning and management of the peri-urban interface: perspectives on an emerging field. Environment and Urbanization, 15(1), 135-147. doi: $10.1177 / 095624780301500103$

Anas, A., Arnott, R. \& Small, K. A. (1998). Urban spatial structure. Journal of Economic Literature, 36(3), 1426-1464. http://www.jstor.org/stable/2564805

Anderson, N. B. \& Bogart, W. T. (2001). The structure of sprawl. Identifying and characterizing employment centers in polycentric metropolitan areas. American Journal of Economics and Sociology, 60(1), 147-169.

Angel, S., Parent, J. \& Civco D. (2007). Urban sprawl metrics: An analysis of global expansion using GIS. ASPRS (American Society of Photogrammetry and Remote Sensing), 2007 Annual Conference. Tampa, Florida.

Audirac, I., Shermyen, A. H. \& Smith, M. T. (1990). Ideal urban form and visions of the good life: Florida's growth management dilemma. Journal of the American Planning Association, 56(4), 470-482.

Balsameda, M., San Martín, I. \& Sebastián, M. (2002). Una aproximación cuantitativa a la "burbuja inmobiliaria”. Situación inmobiliaria (diciembre), Servicio de Estudios de BBVA, 22-28.

Beavestock, J., Smith, R. \& Taylor, P. (2000). World-city network: A new metageography? Annals of the Association of American Geographers, 90(1), 123-134. doi: 10.1111/0004-5608.00188

Berry, B. (Ed.). (1976). Urbanization and Counterurbanization. (Urban Affairs Annual Reviews, vol. 11). Beverly Hills, CA: Sage Publications.

Bertaud, A. \& Malpezzi, S. (1999). The spatial distribution of population in 35 world cities: The role of markets, planning, and topography. Wisconsin-Madison CULER working papers 01-03, University of Wisconsin, Center for Urban Land Economic Research.

Breslaw, J. A. (1990). Density and urban sprawl: A comment. Land Economics, 66(4), 464-469.

Brueckner, J. K. \& Fansler, D. A. (1983). The economics of urban sprawl: Theory and evidence on the spatial sizes of cities. The Review of Economics and Statistics, 65(3), 479-482. http://www. jstor.org/stable/1924193 
Brueckner, J. K. (1997). Reducing the need to travel. Environment and Planning B: Planning and Design, 24(3), 437-499.

Brueckner, J. K. (2000). Urban sprawl: Lessons from Urban Economics. En W. G. Gale \& J. R. Pack (Eds.), Brookings-Wharton Papers on Urban Affairs (pp. 65-89). Washington D.C.: Brookings Institution.

Burchell, R. W., Shad, N. A., Listokin, L., Phillips, H., Downs, A., Seskin, S. (...) \& ECONorthwest. (1998). Costs of sprawl - revisited. Washington, D.C.: National Academy Press.

Burchfield, M., Overman, H. G., Puga, D. \& Turner, M. A. (2006). Causes of sprawl: A portrait from the space. The Quarterly Journal of Economics, 121(2), 587-633.

Cabré, A. \& Módenes, J. A. (1997). Dinàmiques demogràiques recents a la Regió Metropolitana de Barcelona. Revista Económica de Catalunya, 33, 66-75.

Cairncross, F. (1997). The Death of Distance: How the communications revolution will change our lives. Boston, MA: Harvard Business School.

Camagni, R., Gibelli, M. C. \& Rigamonti, P. (2002). Urban mobility and urban form: The social and environmental costs of different patterns of urban expansion. Ecological Economics, 40(2),199-216.

Carrera Alpuente, J. M. (2002). Aproximacions a l'estructura espacial de l'Àrea Metropolitana de Barcelona. Diferents maneres d'ocupar el territori. Papers, n. ${ }^{\circ}$ 36: Regió Metropolitana de Barcelona : Territori, estratègies, planejament (pp. 9-24). Barcelona : Institut d'Estudis Metropolitans de Barcelona.

Carreras Quilis, J. M. (2002). La redistribució de la ciutat al territori de la Regió Metropolitana de Barcelona. Papers n. ${ }^{\circ}$ 36: Ciutat compacta, ciutat difusa (pp. 25-48). Barcelona: Institut d'Estudis Metropolitans de Barcelona.

Carruthers, J. I. \& Ulfarsson, C. F. (2003). Urban sprawl and the cost of public services. Environment and Planning B: Planning and Design, 30(4), 503-522.

Castells, M. (1989). The informational city. Oxford: Basil Blackwell.

Charney, I. (2005). Re-examining suburban dispersal: Evidence from suburban Toronto. Journal of Urban Affairs, 27(5), 467-484. doi: 10.1111/j.0735-2166.2005.00248.x

Chin, N. (2002). Unearthing the roots of urban sprawl: A critical analysis ofform, function and methodo$\log y$. Paper n. ${ }^{\circ}$ 47. Centre for Advanced Spatial Analysis, University College, Londres.

Coffey, W. J. \& Shearmur, R. (2002). Agglomeration and dispersion of high-order service employment in the Montreal Metropolitan Region, 1981-96. Urban Studies, 39(3), 359-378. doi: 10.1080/00420980220112739

Couch, C., Karecha, J., Nuissl, H. \& Rink, D. (2005). Decline and sprawl: An evolving type of urban development observed in Liverpool and Leipzig. European Planning Studies, 13(1), 117-136. doi: http://dx.doi.org/10.1080/0965431042000312433

Dear, M. \& Flusty, S. (1998). Postmodern urbanism. Annals of the Association of American Geographers, 88(1), 50-72. doi: 10.1111/1467-8306.00084

Domene, E. \& Saurí, D. (2006). Urbanization and class-produced natures. Vegetable gardens in the Barcelona Metropolitan Region. Geoforum, 38(2), 287-298.

Domene, E., Saurí, D. \& Parés, M. (2005). Urbanization and sustainable resource use: The case of garden watering in the Metropolitan Region of Barcelona. Urban Geography, 26(6), 520-533. 
Downs, A. (1999). Some realities about sprawl and urban decline. Housing Policy Debate, 10(4), 955974.

Durà-Guimerà, A. (2003). Population deconcentration and social restructuring in Barcelona, a European Mediterranian city. Cities, 20(6), 387-394. doi:10.1016/j.cities.2003.08.004

European Environment Agency. (1999). Environment in the European Union at the turn of the century. Environmental Assessment Report 2. http://www.eea.europa.eu/publications/92-9157-202-0

European Environment Agency. (2002a). Towards an urban atlas. Assessment of spatial data on 25 European cities and urban areas. Environmental Issue Report 30. http://www.eea.europa. eu/publications/environmental_issue_report_2002_30

European Environment Agency. (2002b). Environmental signals 2002. Benchmarking the millennium. Environmental Assessment Report 9. http://www.eea.europa.eu/publications/environmental_assessment_report_2002_9

European Environment Agency. (2006). Urban sprawl in Europe. European Environment Agency Report 10.

Ewing, R. (1997). Is Los Angeles-style sprawl desirable? Journal of American Planning Association, 63(1), 107-126.

Ewing, R., Pendall, R. \& Chen, D. (2002). Measuring Sprawl and its Impact. Washington D.C.: Smart Growth America.

Fernández Durán, R. (2006). El tsunami urbanizador español y mundial. Boletin $C F+S>38 / 39$ : Arquitectura del siglo XXI: más allá de Kioto. http://habitat.aq.upm.es/boletin/n38/arfer. html.

Ferrer, A. (2003). Del crecimiento disperso a la ciudad razonablemente compacta. En A. Font (Ed.), Planeamiento urbanistico. De la controversia a la renovación (pp. 151-168). Barcelona: Diputació de Barcelona, Xarxa de Municipis.

Fishman, R. (1998). Beyond Utopia: Urbanism after the end of cities. En P. Subirós (Ed.), Ciutat real, ciutat idea (pp. 29-36). Barcelona: Center de Cultura Contemporànea de Barcelona.

Folch, R. (1998). Socioecologia i gestió ambiental a làrea de Barcelona. Revista Económica de Catalunya, 34, 57-62.

Font, A. (2007). Planeamiento urbanístico: de la controversia a la renovación. Barcelona: Diputació de Barcelona, Xarxa de Municipis.

Font, A., Llop, C. \& Vilanova, J. M. (1999). La construcció del territori metropolità. Morfogènesi de la regió urbana de Barcelona. Barcelona: Area Metropolitana de Barcelona, Mancomunitat de Municipis.

Font, A., Mas, S., Maristany, L., Carreras, J. M. \& Valls, J. (2005). Transformacions urbanitzadores. Area Metropolitana i Regió Metropolitana. Barcelona: Àrea Metropolitana de Barcelona, Mancomunitat de Municipis.

Frey, H. (1999). Designing the City. Towards a more sustainable urban form. Londres: Spon Press.

Gaja, F. (2008). El “tsunami urbanizador” en el litoral mediterráneo. El ciclo de hiperproducción inmobiliaria 1996-2006. X Coloquio Internacional de Geocrítica. Diez años de cambio en el mundo, en la geografía y en las ciencias sociales 1999-2008. Barcelona, Universidad de Barcelona. Publicado en Scripta Nova, Revista Electrónica de Geografía y Ciencias Sociales (Universidad de Barcelona), 12(270), 1 de agosto de 2008. http://www.ub.edu/geocrit/sn/ 
sn-270/sn-270-66.htm

Galster, G., Hanson, R., Ratcliffe, M., Wolman, H. \& Freihage, J. (2001). Wrestling sprawl to the ground: Defining and measuring an elusive concept. Housing Policy Debate, 12, 681-717.

García, D. \& Riera, P. (2003). Expansion versus density in Barcelona. A valuation exercise. Urban Studies, 40(10), 1925-1936. doi: 10.1080/0042098032000116040

Garcia-López, M. A. \& Muñiz, I. (2007). ¿Policentrismo o dispersión? Una aproximación desde la Nueva Economía Urbana. Investigaciones Regionales, 11, 25-43.

Garcia-López, M. A. \& Muñiz, I. (2010). El impacto espacial de las economías de aglomeración y su efecto sobre la estructura espacial del empleo. El caso de la industria en Barcelona, 19861996. Revista de Economía Aplicada, 18(52), 91-119. http://www.staragon.com/revecap/ revista/numeros/52/pdf/garcia_muniz.pdf

Garcia-López, M. A. (2006). Estructura espacial del empleo y economías de aglomeración: El caso de la industria en la Región Metropolitana de Barcelona. Tesis Doctoral. Departament d'Economia Aplicada, Universitat Autònoma de Barcelona.

Garcia-López, M. A. (2008). Quince años de suburbanización en la Barcelona Metropolitana. ¿Se está dispersando la población? Investigaciones Económicas, 32, 53-86.

Garcia-López, M. A. (2010). Population suburbanization in Barcelona, 1991-2005: Is its spatial structure changing? Journal of Housing Economics, 19(2), 119-132. doi:10.1016/j. jhe.2010.04.002

Glaeser, E. L., Kahn, M. E. (2004). Sprawl and urban growth. En J. V. Henderson, P. Nijkamp, E. S. Mills, P. C. Cheshire \& J. F. Thisse (Eds.), Handbook of Regional and Urban Economics (vol. 4, pp. 2481-2527). Amsterdam: Elsevier.

Goffette-Nagot, F. (2000). Urban spread beyond the city edge. En M. J. Huriot \& J. F. Thisse (Eds.), Economics of cities (pp. 318-340). Cambridge, MA: Cambridge University Press.

Gordon, P. \& Richardson, H. W. (1996). Beyond polycentricity. The dispersed metropolis, Los Angeles, 1970-1990. Journal of the American Planning Association, 62(3), 289-295. doi: 10.1080/01944369608975695

Gordon, P. \& Richardson, H. W. (1997). Are compact cities a desirable planning goal? Journal of the American Planning Association, 63(1), 89-106. doi:10.1080/01944369708975727

Gordon, P. \& Wong, H. L. (1985). The costs of urban sprawl: Some new evidence. Environment and Planning A, 17(5), 661-666.

Graham, S. \& Marvin, S. (2006). Telecommunications and the City. Electronic spaces, urban places. Londres: Routledge.

Gravagnuolo, B. (1998). Historia del urbanismo en Europa 1750-1960. Madrid: Ediciones Akal.

Hall, P. (1996). Ciudades del mañana. Historia del urbanismo en el siglo XX. Barcelona: Ediciones del Serbal.

Hess, G. R., Daley, S. S., Dennison, B. K., McGuin, R. P., Morin, V. Z., Shelton, W. G. (...) \& Wrege, B. M. (2001). Just, what is sprawl anyway? Raleigh, NC: North Carolina State University.

Iglesias, F. (Ed.). (2007). Urbanismo y democracia. Alternativas para evitar la corrupción. Madrid: Fundación Alternativas.

Indovina, F. (1990). La città diffusa. Venezia: Dipartamento di Analisi Economica e Sociale del Territorio, Istituto Universitario di Architettura di Venezia.

Jiménez, F. (2008). Boom urbanístico y corrupción política en España. Mediterráneo Económico, 14 
(número dedicado a: Modernidad, crisis y globalización: problemas de política y cultura, coord. por V. M. Pérez Díaz), 263-285.

Kahn, M. E. (2000). The environmental impact of suburbanization. Journal of Policy Analysis and Management, 19(4), 569-586.

Kahn, M. E. (2006). The Quality of Life in Sprawled versus Compact Cities. Documento preparado para la Mesa Redonda de la OCDE (Organización para la Cooperación Económica y el Desarrollo), Berkeley, CA, marzo 2006.

Kasanko, M., Barredo, J. I., Lavalle, C., McCormick, N., Demicheli, L., Sagres, V. \& Brezger, A. (2006). Are European cities becoming dispersed? A comparative analysis of 15 European urban areas. Landscape and Urban Planning, 77(1-2), 111-130. http://dx.doi.org/10.1016/j. landurbplan.2005.02.003

Kirby, A. (2004). On 'sprawl'. Cities, 21(5), 369-370.

Ladd, H. (1992). Population growth, density and the costs of providing public services. Urban Studies, 29(2), 273-295. doi: 10.1080/00420989220080321

Lang, R. E. \& LeFurgy, J. (2003). Edgeless cities: Examining the non-centered metropolis. Housing Policy Debate, 14(3), 427-460.

Lee, B. (2007). "Edge" or "edgeless" cities? Urban spatial structure in U.S. metropolitan areas, 1980 to 2000. Journal of Regional Science, 47(3), 479-515. doi: 10.1111/j.1467-9787.2007.00517.x

Lee, J. (2007). Geographic Patterns of Urban Residential Development. Kent, OH: Department of Geography, Kent State University.

Lopez, R. \& Hynes, H. P. (2003). Sprawl in the 1990's: Measurement, distribution, and trends. Urban Affairs Review, 38(3), 325-355. doi: 10.1177/1078087402238805

Mallarach, J. M. \& Comas, E. (2005). Bones pràctiques d'incorporació del paisatge en el planejament urbanístic de Catalunya. Elements de Debat Territorial, 22. Barcelona: Diputació de Barcelona, Xarxa de Municipis.

Malpezzi, S. \& Guo, W. K. (2001). Measuring "sprawl". Alternative measures of urban form in U.S. metropolitan areas. Madison, WI: The Center for Urban Land Economics Research, University of Wisconsin.

Marull, J. \& Mallarach, J. M. (2002). La conectividad ecológica en el Área Metropolitana de Barcelona. Ecosistemas, 11(2), s/p.

Ministerio de Fomento. (2006). Cambios de ocupación de suelo en España. Implicaciones para la sostenibilidad. Estudio realizado a partir del Proyecto Corine Land Cover. Madrid: Observatorio de la Sostenibilidad en España (OSE).

Miralles, C. (1997). Transport i ciutat. Reflexió sobre la Barcelona Metropolitana. Bellaterra: Servei de Publicacions de la Universitat Autònoma de Barcelona (UAB).

Monclús, F. J. (1998a). Suburbanización y nuevas periferias. Perspectivas geográfico-urbanísticas. En F. J. Monclús (Ed.), La ciudad dispersa. Suburbanización y nuevas periferias (pp. 5-16). Barcelona: Center de Cultura Contemporànea de Barcelona.

Monclús, F. J. (1998b). Estrategias urbanísticas y crecimiento suburbano en las ciudades españolas: el caso de Barcelona. En F. J. Monclús (Ed.), La ciudad dispersa. Suburbanización y nuevas periferias (pp. 143-168). Barcelona: Center de Cultura Contemporànea de Barcelona.

Muñiz, I. \& Galindo, A. (2005). Urban form and the ecological footprint of commuting. The case of Barcelona. Ecological Economics, 55(4), 499-514. http://dx.doi.org/10.1016/j.ecolecon.2004.12.008 
Muñiz, I. \& Roig, J. L. (2001). Suburbanizacion, distribución espacial de la renta y segregación residencial en la región metropolitana de Barcelona. Documento de trabajo. Departament d'Economia Aplicada, Universitat Autònoma de Barcelona (UAB), 15.

Muñiz, I., Calatayud, D. \& Garcia-López, M. A. (2006). Sprawl. Causas y efectos de la dispersión urbana. Documento de trabajo (Departament d'Economia Aplicada, Universitat Autònoma de Barcelona, UAB), 0603.

Muñiz, I., Galindo, A. \& Garcia-López, M. A. (2003). Cubic spline population density functions and satellite cities delimitation: the case of Barcelona. Urban Studies, 40(7), 1303-1321. doi:10.1080/0042098032000084613

Muñiz, I., Garcia-López, M. A. \& Galindo, A. (2008). The effect of employment sub-centres on population density in Barcelona. Urban Studies, 45(3), 627-649. doi:10.1177/0042098007087338

Muñoz, F. (2003). Lock living: Urban sprawl in Mediterranean cities. Cities, 20(6), 381-385. doi: http://dx.doi.org/10.1016/j.cities.2003.08.003

Muñoz, F. (2005). La producció residencial de baixa densitat. Elements de Debat Territorial, 21. Barcelona: Diputació de Barcelona, Xarxa de Municipis.

Muñoz, F. (2008). Urbanalización. Paisajes comunes, lugares globales. Barcelona: Gustavo Gili Ed.

Naredo, J. M. (1996). La burbuja inmobiliario-financiera en la coyuntura económica reciente (19851995). Madrid: Siglo XXI de España Editores.

Naredo, J. M., Carpintero, O. \& Marcos, C. (2003). Los aspectos patrimoniales en la coyuntura económica actual: Nuevos datos e instrumentos de análisis. Cuadernos de Información Económica, $171,26-56$.

Nel.lo, O. (1999). Reflexiones sobre el futuro de la ciudad. En A. García-Espuche \& S. Rueda (Eds.), La ciudad sostenible (pp. 13-45). Barcelona: Center de Cultura Contemporànea de Barcelona.

Nel.lo, O. (2002). Cataluña, ciudad de ciudades. Lleida: Editorial Milenio.

Newman, P. \& Kenworthy, J. (1989). Cities and Automobile Dependence: A Sourcebook. Aldershot, UK: Gower.

Newman, P. \& Thornley, A. (1996). Urban Planning in Europe: International competition, national systems and planning projects. Londres: Routledge.

Ogawa, H. \& Fujita, M. (1980). Equilibrium land use patterns in a non-monocentric city. Journal of Regional Science, 20, 455-475.

Paül, V. \& Tonos, M. (2005). Containing urban sprawl: Trends in land use and spatial planning in the Metropolitan Region of Barcelona. Journal of Environmental Planning and Management, 48(1), 7-35. doi: 10.1080/0964056042000308139

Peiser, R. (1990). Density and urban sprawl: A reply. Land Economics, 66(4), 469-473.

Persky, J. \& Wiewel, W. (2000). When corporates leave town. Detroit, Il: Wayne State University Press.

Pfister, N., Freestone, R. \& Murphy, P. (2000). Polycentricity or dispersion? Changes in center employment in metropolitan Sydney, 1981 to 1996. Urban Geography, 21(5), 428-442. doi 10.2747/0272-3638.21.5.428

Power, A. (2001). Social exclusion and urban sprawl: Is the rescue of the cities possible? Regional Studies, 35(8), 731-742. doi: 10.1080/00343400120084713

Pressman, N. (1985). Forces for spatial change. En J. Brotchie, P. Newton, P. Hall \& P. Nijkamp (Eds.), The Future of Urban Form. The impact of new technology (pp. 349-361). Londres: Routledge. 
Razin, E. \& Rosentraub, M. (2000). Are fragmentation and sprawl interlinked? North American evidence. Urban Affairs Review, 35(6), 821-836.

Roca, J., Burns, M. C. \& Carreras, J. M. (2004). Monitoring Urban Sprawl around Barcelona's Metropolitan Area with the Aid of Satellite Imagery. Ponencia preparada para el $20^{\circ}$ Congreso de International Society for Photogrammetry and Remote Sensing (ISPRS), Estambul, 12 a 23 de julio de 2004 .

Rogers, R. \& Gumuchdjian, P. (2000). Ciudades para un pequeño planeta. Barcelona: Gustavo Gili. Romero, J. (2009). Geopolítica y gobierno del territorio en España. Valencia: Ed. Tirant lo Blanch.

Rueda, S. (1998). Periurbanización y complejidad en los sistemas urbanos. En F. J. Monclús (Ed.), La ciudad dispersa. Suburbanización y nuevas periferias (pp. 83-110). Barcelona: Center de Cultura Contemporànea de Barcelona.

Rueda, S. (2002). Els costos ambientals dels models urbans dispersos. Revista Papers (Institut d ${ }^{1}$ Estudis Metropolitans, Barcelona), 36, 73-104.

Serra, J. (1997). La Barcelona metropolitana: Transformacions generades per l'ocupació residencial. Revista Económica de Catalunya, 33, 77-88.

Serratosa, A. (1998). El Pla Territorial Metropolità de Barcelona i el dinamisme econòmic. Revista Econòmica de Catalunya, 34, 89-95.

Serratosa, A. (1999). Ciutat compacta, urbanització dispersa. En A. García-Espuche \& S. Rueda (Eds.), La ciutat sostenible (pp. 47-54). Barcelona: Center de Cultura Contemporànea de Barcelona.

Shearmur, R. \& Alvergne, C. (2003). Regional planning policy and the location of employment in the Ile-de-France. Does policy matter? Urban Affairs Review, 39(1), 3-31. doi: $10.1177 / 1078087403253557$

Shearmur, R. \& Coffey, W. J. (2002). A tale of four cities: Intrametropolitan employment distribution in Toronto, Montreal, Vancouver and Ottawa-Hull 1981-1996. Environment and Planning A 34(4), 575-598.

Shearmur, R., Coffey, W., Dubé, C. \& Barbonne, R. (2007). Intrametropolitan employment structure: Polycentriciy, scatteration, dispersal and chaos in Toronto, Montreal and Vancouver, 1996-2001. Urban Studies, 44(9), 1713-1738. doi: 10.1080/00420980701426640

Short, J. R., Hanlon, B. \& Vicini, T. J. (2007). The decline of inner suburbs; The new suburban gothic in the United States. Geography Compass, 1(3), 641-656. doi: 10.1111/j.17498198.2007.00020.x

Solans, J. A. (1998). L’ordenació del sistema urbà central de Catalunya. Revista Económica de Catalunya, 34, 84-88.

Solans, J. A. (2002). L’ocupació del sòl en el sistema metropolità central durant el periode 1980-1998. Revista Papers (Institut d ${ }^{1}$ Estudis Metropolitans, Barcelona), 36, 49-72.

Solé, A. \& Viladecans, E. (2007). Economic and Political Determinants of Urban Expansion: Exploring the local connection. Seminari de la Xarxa de Referència d'R+D+I en Economia Aplicada (XREAP), 17 de desembre, Universitat de Barcelona.

Song, Y. \& Knaap, G. J. (2004). Measuring urban form. Journal of the American Planning Association, 70(2), 210-225. doi:10.1080/01944360408976371

Sullivan, A. M. (1986). A general equilibrium model with agglomerative economies and decentralized employment. Journal of Urban Economics, 20(1), 55-75. http://dx.doi.org/10.1016/00941190(86)90015-X 
Torrens, P. M. \& Alberti, M. (2000). Measuring Sprawl. CASA Working Paper 27, Center for Advanced Spatial Analysis (CASA), University College London.

Trilla, C. (2001). La politica d'habitatge en una perspectiva europea comparada. Col.lecció Estudis Socials, n. ${ }^{\circ}$ 9. Barcelona: Fundació La Caixa.

Trilla, C. (1997). La Barcelona metropolitana: Transformacions generades per l'ocupació residencial. Revista Económica de Catalunya, 33, 105-115.

Tsai, Y. H. (2005). Quantifying urban form: Compactness versus 'sprawl'. Urban Studies, 42(1), 141 161. doi: $10.1080 / 0042098042000309748$

Vecslir, L. (2007). Paisajes de la nueva centralidad. Urban, 12, 34-55.

Wassmer, R. W. (2000). Urban Sprawl in a U.S. Metropolitan Area: Ways to measure and a comparison of the Sacramento areas in California and the U.S. Sacramento, CA: Department of Public Policy and Administration, California State University.

Wassmer, R. W. (2001). An Economist's Perspective on Urban Sprawl: With an application on metropolitan areas in the American West. Sacramento, CA: Department of Public Policy and Administration, California State University.

Wassmer, R. W. \& Edwards, D. (2005). Causes of Urban Sprawl (decentralization) in the United States: Natural evolution, fight from the blight and fiscalization of land use. Sacramento, CA: Department of Public Policy and Administration, California State University.

Wheller, J., Aoyama, Y. \& Warf, B. (2000). City Space, Industrial Space, and Cyberspace: Cities in the telecomunications age. The fracturing geographies. Londres: Routledge.

White, M. J. (1999). Urban areas with decentralized employment: Theory and empirical work. En E. S. Mills \& P. Cheshire (Eds.), Handbook of Regional and Urban Economics (vol. 3, pp. 1375-1412). Amsterdam: Elsevier.

Whyte, W. H., Jr. (1958). Urban sprawl. En W. H. Whyte, Jr (Ed.), The Exploding Metropolis (pp. 115-139). Garden City, NY: Doubleday and Co.

Wolman, H., Galster, G., Hanson, R., Ratcliffe, M. \& Furdell, K.. (2002). Measuring Sprawl: Problems and solutions. 2002 Meeting of the Association of Collegiate Schools of Planning, Baltimore.

Yeh, A. G. O. \& Li, X. (1998). Sustainable land development model for rapid growth areas using GIS. International Journal of Geographical Information Science, 12(2), 169-189. doi: $10.1080 / 136588198241941$

Yeh, A. G. O. \& Li, X. (2001). Measurement and monitoring of urban sprawl in a rapidly growing region using entropy. Photogrammetric Engineering and Remote Sensing, 67(1), 83-90. 
